ESAIM: M2AN

Vol. 40, No 2, 2006, pp. 239-267

DOI: $10.1051 / \mathrm{m} 2 \mathrm{an}: 2006010$
ESAIM: Mathematical Modelling and Numerical Analysis

www.edpsciences.org/m2an

\title{
AN EQUILIBRATED RESIDUAL METHOD WITH A COMPUTABLE ERROR APPROXIMATION FOR A SINGULARLY PERTURBED REACTION-DIFFUSION PROBLEM ON ANISOTROPIC FINITE ELEMENT MESHES
}

\author{
SERGey Grosman ${ }^{1}$
}

\begin{abstract}
Singularly perturbed reaction-diffusion problems exhibit in general solutions with anisotropic features, e.g. strong boundary and/or interior layers. This anisotropy is reflected in a discretization by using meshes with anisotropic elements. The quality of the numerical solution rests on the robustness of the a posteriori error estimator with respect to both, the perturbation parameters of the problem and the anisotropy of the mesh. The equilibrated residual method has been shown to provide one of the most reliable error estimates for the reaction-diffusion problem. Its modification suggested by Ainsworth and Babuška has been proved to be robust for the case of singular perturbation. In the present work we investigate the modified method on anisotropic meshes. The method in the form of Ainsworth and Babuška is shown here to fail on anisotropic meshes. We suggest a new modification based on the stretching ratios of the mesh elements. The resulting error estimator is equivalent to the equilibrated residual method in the case of isotropic meshes and is proved to be robust on anisotropic meshes as well. Among others, the equilibrated residual method involves the solution of an infinite dimensional local problem on each element. In practical computations an approximate solution to this local problem was successfully computed. Nevertheless, up to now no rigorous analysis has been done showing the appropriateness of any computable approximation. This demands special attention since an improper approximate solution to the local problem can be fatal for the robustness of the whole method. In the present work we provide one of the desired approximations. We prove that the method is not affected by the approximate solution of the local problem.
\end{abstract}

Mathematics Subject Classification. 65N15, 65N30, 65N50.

Received: October 18, 2004.

\section{INTRODUCTION}

Let $\Omega \subset \mathbb{R}^{2}$ be an open domain with polyhedral boundary $\partial \Omega$. Consider the reaction-diffusion problem with homogeneous Dirichlet boundary conditions

$$
-\Delta u+\kappa^{2} u=f \text { in } \Omega, \quad u=0 \text { on } \partial \Omega,
$$

where $\kappa$ is a non-negative constant.

\footnotetext{
Keywords and phrases. A posteriori error estimation, singular perturbations, reaction-diffusion problem, robustness, anisotropic solution, stretched elements.

${ }^{1}$ Institut für Mathematik und Bauinformatik, Fakultät für Bauingenieur- und Vermessungswesen, Universität der Bundeswehr München, 85577 Neubiberg, Germany. sergey.grosman@unibw-muenchen.de
} 
If $\kappa \gg 1$, then we have a singularly perturbed problem. Singularly perturbed problems arise in many fields of application. For instance boundary value problems formulated on thin domains [26], where $\kappa$ is proportional to the inverse of the domain thickness. They also arise in mathematical models of physical problems, where diffusion is small compared with reaction and convection.

Such problems yield solutions with local anisotropic behavior, e.g. boundary and/or interior layers. In these cases a special mesh adaptivity is desirable. Triangles should not only adapt in size but also in shape, in order to fit the function to be approximated better. Standard finite element meshes consist of isotropic elements. In this work we investigate so-called anisotropic elements. They are characterized by a large aspect ratio (the ratio of the diameters of the circumscribed and inscribed circles). The singularly perturbed reaction diffusion problem typically requires triangles stretched along the boundary or in the direction of the interior layer $[4,5,8]$.

There is a large variety of a posteriori error estimation techniques. We do not aim at giving an overview of the related works here, instead we refer to $[3,25]$ and citations therein. Some types of a posteriori error estimation methods have already been generalized for anisotropic meshes. They include the works on residual error estimators [18,20,22], hierarchical error estimators [13,14] and Dirichlet local problem error estimators [19,21]. The modified equilibrated residual method [1] has been shown to provide one of the most reliable error estimates for the singularly perturbed reaction-diffusion problem. We will refer to this method as Ainsworth-Babuška estimator. The equilibrated residual method dates back to [11] and was further developed in [1,2, 10, 23]. The main purpose of the current work is to consider the estimator from [1] on anisotropic meshes and to construct upper and lower error bounds. It turns out that the equilibrated residual method fails on anisotropic meshes due to a (potentially unbounded) factor appearing in the lower bound. This factor is $O(1)$ on isotropic meshes, but it can be of size of the maximum aspect ratio on anisotropic meshes. We propose a new modification for anisotropic elements leading to a robust error estimator. The upper error bound of the modification contains an alignment measure which is in accordance with the results by Kunert [21].

Among others, the robustness of the equilibrated residual method relies on the solution of a local residual problem on each element. This problem is infinite dimensional and does not allow an exact solution in general. In practical computations it is therefore solved approximately by means of the finite element method. According to the existing theory, however, the equilibrated residual method is guaranteed to be robust if the exact solution to the local problem can be computed. The potentially fatal role of the approximation of this solution has been poorly investigated up to now, which gives rise to heavy criticism of the whole method. In this work we provide an appropriate basis for the solution of the local problem and show that the whole method is not affected by this approximation.

The paper is organized as follows. After describing the model problem and its discretization in Section 2, we give a brief overview of the standard equilibrated residual method and its modification for the singularly perturbed case in Section 3. Moreover we derive the upper error bound. In Section 4 some properties of the equilibrated residual method on anisotropic meshes are proved. In Section 5 the lower error bound is given with the standard estimator of the modified equilibrated residual method [1]. Furthermore, we introduce in Section 6 the modification of the equilibrated residual method for the anisotropic case and prove that the resulting estimator is robust. In Section 7 a suitable approximate solution of the local problem is proposed and its equivalence to the exact solution is shown. At the end of Section 7 the computable error estimator is defined and the resulting error bounds are stated. Finally, a numerical example and some additional remarks complete the discussion.

\section{The Model PRoblem, its Discretization AND SOME NOTATION}

Let $\omega$ be an open subset of $\Omega \subset \mathbb{R}^{2}$. For any subset $\omega \subset \Omega$ we introduce the usual $L_{2}$ inner product $(u, v)_{L_{2}(\omega)}:=\int_{\omega} u v \mathrm{~d} x$ and inner product $B_{\omega}(u, v):=\int_{\omega}\left(\nabla^{\top} u \nabla v+\kappa^{2} u v\right) \mathrm{d} x$, which lead to the norms $\|v\|_{L_{2}(\omega)}^{2}=(u, u)_{L_{2}(\omega)}$ and $\|v\|_{\omega}^{2}=B_{\omega}(u, u)$. When $\omega=\Omega$, the subscript will be omitted.

We consider problem (1) and assume $f \in L_{2}(\Omega)$. The corresponding variational formulation for (1) is

$$
\text { Find } u \in H_{0}^{1}(\Omega): \quad B(u, v)=(f, v) \quad \forall v \in H_{0}^{1}(\Omega) .
$$


We utilize a family $\mathcal{F}=\{\mathcal{T}\}$ of conforming triangulations $\mathcal{T}$ of $\Omega$, i.e. any two triangles are either disjoint or share a common vertex or an edge. Let $X \subset H_{0}^{1}(\Omega)$ be the space of continuous, piecewise linear functions over $\mathcal{T}$ that vanish on $\partial \Omega$. Then the finite element solution $u_{X} \in X$ is uniquely defined by

$$
B\left(u_{X}, v_{X}\right)=\left(f, v_{X}\right) \quad \forall v_{X} \in X
$$

Due to the Lax-Milgram Lemma both problems (2) and (3) admit unique solutions.

Let $\mathcal{N}$ be the set of all the nodes in the triangulation $\mathcal{T}$, then we denote by $\mathcal{N}(K)$ and $\mathcal{N}(\gamma)$ the sets of all vertices of a triangle $K$ and an edge $\gamma$ respectively. Let $x_{n} \in \mathcal{N}$ be any node and let $\theta_{n}$ be the Lagrange basis function associated with that node. Let $\widetilde{x}_{n}=\operatorname{supp} \theta_{n}$ be the patch of elements around vertex $x_{n}$. Similarly, $\mathcal{E}_{n}$ is the set of those edges which have a vertex at $x_{n}$. For an edge $\gamma$ and an element $K$ of the triangulation $\mathcal{T}$, let $|\gamma|=\operatorname{meas}_{1}(\gamma)$ and $|K|=$ meas $_{2}(K)$ denote the length of $\gamma$ and area of $K$, respectively. In what follows $\omega_{K}$ will denote the patch of elements around $K$ that satisfy $K^{\prime} \subset \omega_{K}$ if and only if $\bar{K} \cap \overline{K^{\prime}}$ is nonempty. Analogously we define the patch $\omega_{\gamma}$ of an edge $\gamma: K \subset \omega_{\gamma}$ if and only if $\gamma \subset \partial K$.

We will need an extension operator $F_{\text {ext }}: \mathbb{P}^{0}(\gamma) \mapsto \mathbb{P}^{0}(K)$ defined by

$$
F_{\text {ext }}(\varphi)(x):=\left.\varphi\right|_{\gamma} \equiv \text { const. }
$$

Bubble functions play an important role in the derivation of lower error bounds. We will define them in the usual way, see for example [24]. Denote by $\lambda_{K, 1}, \lambda_{K, 2}, \lambda_{K, 3}$ the barycentric coordinates of an arbitrary triangle $K$. The element bubble function $b_{K}$ is defined by

$$
b_{K}:=27 \lambda_{K, 1} \lambda_{K, 2} \lambda_{K, 3} \text { on } K
$$

Let $\gamma=\operatorname{int}\left(\bar{K}_{1} \cap \bar{K}_{2}\right)$ be an inner edge of $\mathcal{T}$. Enumerate the vertices of $K_{1}$ and $K_{2}$ such that the vertices of $\gamma$ are numbered first. Define the edge bubble function $b_{\gamma}$ and the edge spline function $s_{\gamma}$ by

$$
\begin{aligned}
b_{\gamma}:=4 \lambda_{K_{i}, 1} \lambda_{K_{i}, 2} & & \text { on } K_{i}, i=1,2 \\
s_{\gamma}:=\frac{18}{\sqrt{3}} \lambda_{K_{i}, 1} \lambda_{K_{i}, 2}\left(\lambda_{K_{i}, 2}-\lambda_{K_{i}, 1}\right) & & \text { on } K_{i}, i=1,2 .
\end{aligned}
$$

For simplicity we assume that $b_{K}, b_{\gamma}$ and $s_{\gamma}$ are extended by zero outside their original domain of definition. It holds that $0 \leq b_{K}(x), b_{\gamma}(x) \leq 1,-1 \leq s_{\gamma}(x) \leq 1$ and $\left\|b_{K}\right\|_{L_{\infty}(K)}=\left\|b_{\gamma}\right\|_{L_{\infty}(K)}=\left\|s_{\gamma}\right\|_{L_{\infty}(K)}=1$.

We use the abbreviation $a \preceq b$ for $a \leq C b$, with a positive constant $C$ independent of $a$ and $b$. We also write $a \sim b$ when $a \preceq b$ and $a \succeq b$. All constants used in this work are independent of $h, \kappa$ and the aspect ratio of the elements.

\section{The Equilibrated Residual Method}

In this section a brief overview over the equilibrated residual method is given since we strongly require parts of this method for our subsequent analysis. The equilibrated residual method may be found in [3] and its modification for the singularly perturbed case in [1].

\subsection{The equilibrated residual method}

Consider the model problem of Section 2. Then the error $e:=u-u_{X}$ belongs to the space $H_{0}^{1}(\Omega)$ and satisfies the variational formulation

$$
B(e, v)=B(u, v)-B\left(u_{X}, v\right)=(f, v)-B\left(u_{X}, v\right) \quad \forall v \in H_{0}^{1}(\Omega) .
$$

For an element $K$ with boundary $\partial K$, let $n_{K}$ be the outer normal vector. Next we introduce a set of boundary fluxes $\left\{g_{K}: K \in \mathcal{T}\right\}$ which approximate the actual fluxes of the exact solution on the element boundaries, 
$\left.g_{K} \approx n_{K} \cdot \nabla u\right|_{K}$. Taking into account that the trace of the true solution is continuous on the edges, we construct the approximate fluxes $g_{K}$ so that the condition

$$
g_{K}+g_{K^{\prime}}=0 \text { on } \partial K \cap \partial K^{\prime}, \quad K, K^{\prime} \in \mathcal{T}
$$

holds true. With this definition the residual on the right hand side of (4) can be decomposed into contributions from the individual elements

$$
(f, v)-B\left(u_{X}, v\right)=\sum_{K \in \mathcal{T}}\left\{(f, v)_{K}-B_{K}\left(u_{X}, v\right)+\int_{\partial K} g_{K} v \mathrm{~d} s\right\} \quad \forall v \in H_{0}^{1}(\Omega) .
$$

The term in parentheses defines a linear functional on the space of the locally admissible functions

$$
V_{K}=\left\{v: v=\left.w\right|_{\Omega} \text { for some } w \in H_{0}^{1}(\Omega)\right\} .
$$

If the parameter $\kappa$ in (1) is not zero, there is a unique solution $\phi_{K} \in V_{K}$ to the local residual problem

$$
B_{K}\left(\phi_{K}, v\right)=(f, v)_{K}-B_{K}\left(u_{X}, v\right)+\int_{\partial K} g_{K} v \mathrm{~d} s \quad \forall v \in V_{K} .
$$

If $\kappa$ vanishes then the problem will have a solution if and only if the collection of fluxes $\left\{g_{K}: K \in \mathcal{T}\right\}$ satisfies the so-called equilibration condition

$$
0=(f, 1)_{K}-B_{K}\left(u_{X}, 1\right)+\int_{\partial K} g_{K} \mathrm{~d} s
$$

This condition means that the boundary flux $g_{K}$ is in equilibrium with the interior load. Note that the local problem (7) is infinite dimensional. The solution $\phi_{K}$ is treated as an approximation of the true error on the element $K$. It yields the a posteriori error estimation $\left\|e\left|\left\|^{2} \sim \sum_{K \in \mathcal{T}}\right\| \phi_{K}\right|\right\|_{K}^{2}$, which will be shown later.

The substitution of (7) into (6) implies

$$
B(e, v)=(f, v)-B\left(u_{X}, v\right)=\sum_{K \in \mathcal{T}} B_{K}\left(\phi_{K}, v\right), \text { for all } v \in V .
$$

An immediate consequence of this result is the upper bound on the true error. We obtain from the CauchySchwarz inequality that

and conclude that

$$
|B(e, v)| \leq \sum_{K \in \mathcal{T}}\left\|\phi_{K}\right\|\left\|_{K} \mid\right\| v\left\|_{K} \leq\left\{\sum_{K \in \mathcal{T}}\left\|\phi_{K}\right\|_{K}^{2}\right\}^{1 / 2}\right\| v\|\|,
$$

$$
\|\mid\| \sup _{v \in H_{0}^{1}(\Omega):\|v\| \|=1} B(e, v) \leq\left\{\sum_{K \in \mathcal{T}}\left\|\phi_{K}\right\|_{K}^{2}\right\}^{1 / 2} .
$$

These developments lead to the following theorem:

Theorem 3.1 (upper error bound). Let $\left\{g_{K}: K \in \mathcal{T}\right\}$ be any set of boundary fluxes satisfying condition (5). Additionally, if $\kappa$ vanishes, then (8) is assumed to hold on all elements that do not abut the boundary $\partial \Omega$. Then, the global error in the finite element approximation is bounded by

$$
\|e \mid\|^{2} \leq \sum_{K \in \mathcal{T}}\left\|\phi_{K}\right\|_{K}^{2} .
$$

Proof. For the proof see the argumentation above. 


\subsection{Construction of the equilibrated fluxes}

For the construction of the equilibrated fluxes we adopt the theory of [3] which we will briefly repeat in this section. It will be assumed that the finite element subspace $X$ is constructed using linear elements on a partition $\mathcal{T}$ of the domain $\Omega$ into triangular elements. The key issue of the lower bound of the error is the construction of appropriate approximate fluxes. The procedure that will be developed produces a set of fluxes $\left\{g_{K}\right\}$ that satisfy the first-order equilibration conditions:

$$
\left.\begin{array}{rl}
\left(f, \theta_{n}\right)_{K}-B_{K}\left(u_{X}, \theta_{n}\right)+\int_{\partial K} g_{K} \theta_{n} \mathrm{~d} s & =0 \quad \forall n \in \mathcal{N}(K) \\
g_{K}+g_{K^{\prime}} & =0 \text { on } \partial K \cap \partial K^{\prime}
\end{array}\right\}
$$

It is convenient to look for $\left.g_{K}\right|_{\gamma}$ belonging to $\operatorname{span}\left\{\theta_{n}: n \in \mathcal{N}(\gamma)\right\}$ on all edges. Ainsworth and Oden [3] suggest to choose the degrees of freedom for the fluxes to be the moments $\mu_{K, n}^{\gamma}=\int_{\gamma} g_{K} \theta_{n} \mathrm{~d} s$ with respect to the FEM basis functions $\theta_{n}$ associated with $x_{n} \in \mathcal{N}(\gamma)$. Thereby we avoid a global problem by reducing the construction of fluxes to computation of the moments over local patches of elements.

Let $\mathcal{N}(\gamma)=\left\{x_{l}, x_{r}\right\}$, then it can be shown that the actual flux may be reconstructed from its moments:

$$
\left.g_{K}\right|_{\gamma}=\frac{2}{|\gamma|}\left\{\left(2 \mu_{K, l}^{\gamma}-\mu_{K, r}^{\gamma}\right) \theta_{l}+\left(-\mu_{K, l}^{\gamma}+2 \mu_{K, r}^{\gamma}\right) \theta_{r}\right\}
$$

Note that (10) could be rewritten in the form

$$
\left.g_{K}\right|_{\gamma}=\mu_{K, l}^{\gamma} \psi_{l}+\mu_{K, r}^{\gamma} \psi_{r}
$$

where $\psi_{l}$ and $\psi_{r}$ are the dual basis functions corresponding to $\theta_{l}$ and $\theta_{r}$, i.e. $\left(\psi_{i}, \theta_{j}\right)_{L_{2}(\gamma)}=\delta_{i j}$ for $i, j \in\{l, r\}$ :

$$
\psi_{l}=\frac{2}{|\gamma|}\left(2 \theta_{l}-\theta_{r}\right), \quad \psi_{r}=\frac{2}{|\gamma|}\left(-\theta_{l}+2 \theta_{r}\right)
$$

In order to determine the boundary fluxes, it is sufficient to determine the moments of the flux with respect to the basis functions. The first-order equilibration conditions (9) for the flux $g_{K}$ may be rewritten in terms of the flux moments in the form

$$
\left.\begin{array}{llll}
\sum_{\gamma \subset \partial K} \mu_{K, n}^{\gamma}=\Delta_{K}\left(\theta_{n}\right) & & \forall n \in \mathcal{N}(K) & \\
\mu_{K, n}^{\gamma}+\mu_{K^{\prime}, n}^{\gamma}=0 & & \forall n \in \mathcal{N}(\gamma), \quad \gamma=\partial K \cap \partial K^{\prime}
\end{array}\right\}
$$

where

$$
\Delta_{K}\left(\theta_{n}\right)=B_{K}\left(u_{X}, \theta_{n}\right)-\left(f, \theta_{n}\right)_{K}
$$

In (11) we used the convention that $\mu_{K, n}^{\gamma}=0$ if $n \notin \mathcal{N}(\gamma)$.

The conditions in (11) have one of two distinct structures depending on the location of the node $x_{n}$ in the interior or on the boundary of $\Omega$. Here we omit the case of a boundary vertex, see [3] for details. Assume $x_{n}$ to be an interior vertex. The elements and edges are labeled as shown in Figure 1. The moment equilibration conditions (11) for the elements $K \in \widetilde{x}_{n}$ associated with the node $x_{n}$ may be rewritten in the form

$$
\left.\begin{array}{rcc}
\mu_{1, n}^{\gamma_{1}}+\mu_{1, n}^{\gamma_{2}} & = & \Delta_{1}\left(\theta_{n}\right) \\
\mu_{2, n}^{\gamma_{2}}+\mu_{2, n}^{\gamma_{3}} & = & \Delta_{2}\left(\theta_{n}\right) \\
& \vdots & \\
\mu_{N, n}^{\gamma_{N}}+\mu_{N, n}^{\gamma_{1}} & = & \Delta_{N}\left(\theta_{n}\right)
\end{array}\right\}
$$




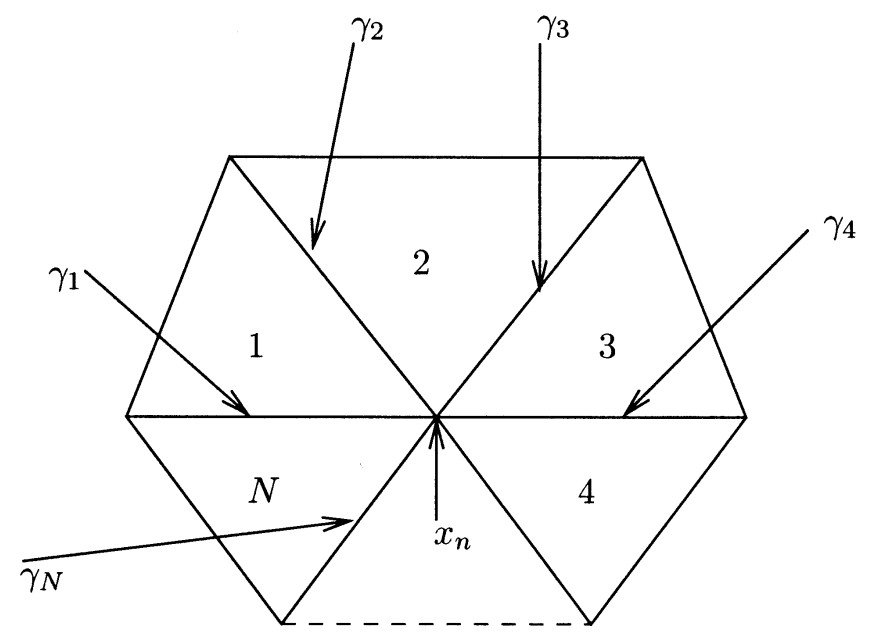

FiguRE 1. The patch of elements influenced by the basis function $\theta_{n}$.

with constraints on the edges

$$
\left.\begin{array}{ccc}
\mu_{1, n}^{\gamma_{1}}+\mu_{N, n}^{\gamma_{1}} & = & 0 \\
\mu_{2, n}^{\gamma_{2}}+\mu_{1, n}^{\gamma_{2}} & = & 0 \\
& \vdots & \\
\gamma_{N, n}^{\gamma_{N}}+\mu_{N-1, n}^{\gamma_{N}} & = & 0
\end{array}\right\} .
$$

It is shown in [3] that this system of $2 N$ equations for $2 N$ unknowns has a one-parametric family of solutions. We recall from [3] which solution should be selected.

The ideal situation would be to choose the flux moments $\left\{g_{K}\right\}$ satisfying $\mu_{K, n}^{\gamma} \approx \int_{\gamma} \theta_{n} n_{K} \cdot \nabla u \mathrm{~d} s$. Since the true fluxes are unknown, the flux moments are selected so that

$$
\mu_{K, n}^{\gamma} \approx \widetilde{\mu}_{K, n}^{\gamma}:=\left.\int_{\gamma} \theta_{n} n_{K} \cdot \nabla u_{X}\right|_{K} \mathrm{~d} s
$$

We seek flux moments that minimize the objective

$$
\frac{1}{2} \sum_{K \in \widetilde{x}_{n}} \sum_{\gamma \subset \partial K}\left(\mu_{K, n}^{\gamma}-\widetilde{\mu}_{K, n}^{\gamma}\right)^{2} .
$$

Introducing Lagrange multipliers we come to the optimality condition. The Lagrangian is given by

$$
\begin{aligned}
& \mathcal{L}\left(\left\{\widetilde{\mu}_{K, n}^{\gamma}\right\},\left\{\lambda_{\gamma}\right\},\left\{\sigma_{K}\right\}\right)=\frac{1}{2} \sum_{K \in \widetilde{x}_{n}} \sum_{\gamma \subset \partial K}\left(\mu_{K, n}^{\gamma}-\widetilde{\mu}_{K, n}^{\gamma}\right)^{2} \\
& +\sum_{K \in \widetilde{x}_{n}} \sigma_{K, n}\left(\Delta_{K}\left(\theta_{n}\right)-\sum_{\gamma \subset \partial K} \mu_{K, n}^{\gamma}\right)+\sum_{\gamma=\partial K \cap \partial K^{\prime}} \lambda_{\gamma, n}\left(\mu_{K, n}^{\gamma}+\mu_{K^{\prime}, n}^{\gamma}\right) .
\end{aligned}
$$

Here we used the convention that $\lambda_{\gamma, n}=0$ on $\gamma \subset \partial \Omega$. We conclude that the conditions for a stationary point consist of two parts; the first part is (11), the second part is

$$
\mu_{K, n}^{\gamma}-\widetilde{\mu}_{K, n}^{\gamma}-\sigma_{K, n}+\lambda_{\gamma, n}=0
$$


Using the second part of (11) we obtain

$$
\lambda_{\gamma, n}= \begin{cases}\frac{1}{2}\left(\sigma_{K, n}+\sigma_{K^{\prime}, n}+\widetilde{\mu}_{K, n}^{\gamma}+\widetilde{\mu}_{K^{\prime}, n}^{\gamma}\right) & \gamma=\partial K \cap \partial K^{\prime} \\ 0 & \gamma=\partial K \cap \partial \Omega\end{cases}
$$

Using the last formula together with (15) the flux moments are expressed as

$$
\mu_{K, n}^{\gamma}= \begin{cases}\frac{1}{2}\left(\sigma_{K, n}-\sigma_{K^{\prime}, n}+\widetilde{\mu}_{K, n}^{\gamma}-\widetilde{\mu}_{K^{\prime}, n}^{\gamma}\right) & \gamma=\partial K \cap \partial K^{\prime} \\ \sigma_{K, n}+\widetilde{\mu}_{K, n}^{\gamma} & \gamma=\partial K \cap \partial \Omega .\end{cases}
$$

Substituting this into the first equation of (11) we obtain the following conditions for $\left\{\sigma_{K, n}: K \in \widetilde{x}_{n}\right\}$ :

$$
\frac{1}{2} \sum_{\gamma=\partial K \cap \partial K^{\prime}}\left(\sigma_{K, n}-\sigma_{K^{\prime}, n}\right)+\sum_{\gamma \subset \partial K \cap \partial \Omega} \sigma_{K, n}=\widetilde{\Delta}_{K}\left(\theta_{n}\right) \quad \forall K \in \widetilde{x}_{n}
$$

where

$$
\begin{gathered}
\widetilde{\Delta}_{K}\left(\theta_{n}\right):=B_{K}\left(u_{X}, \theta_{n}\right)-\left(f, \theta_{n}\right)_{K}-\int_{\partial K}\left\langle\frac{\partial u_{X}}{\partial n_{K}}\right\rangle \theta_{n} \mathrm{~d} s, \\
\left\langle\frac{\partial u_{X}}{\partial n_{K}}\right\rangle:= \begin{cases}\frac{1}{2} n_{K} \cdot\left\{\left(\nabla u_{X}\right)_{K}+\left(\nabla u_{X}\right)_{K^{\prime}}\right\} & \text { on } \partial K \cap \partial K^{\prime} \\
n_{K} \cdot\left(\nabla u_{X}\right)_{K} & \text { on } \partial K \cap \partial \Omega .\end{cases}
\end{gathered}
$$

The conditions (17) form a linear system of equations over the element patches $\widetilde{x}_{n}$ with unknowns $\left\{\sigma_{K, n}: K \in \widetilde{x}_{n}\right\}$ corresponding to the elements in the patch. The specific form for an interior vertex is

$$
\frac{1}{2}\left[\begin{array}{rrlllr}
2 & -1 & & \ldots & & -1 \\
-1 & 2 & -1 & \ldots & & 0 \\
\vdots & & & & & \vdots \\
0 & & \ldots & -1 & 2 & -1 \\
-1 & & \cdots & & -1 & 2
\end{array}\right]\left[\begin{array}{c}
\sigma_{1} \\
\sigma_{2} \\
\vdots \\
\sigma_{N-1} \\
\sigma_{N}
\end{array}\right]=\left[\begin{array}{c}
\widetilde{\Delta}_{1}\left(\theta_{n}\right) \\
\widetilde{\Delta}_{2}\left(\theta_{n}\right) \\
\vdots \\
\widetilde{\Delta}_{N-1}\left(\theta_{n}\right) \\
\widetilde{\Delta}_{N}\left(\theta_{n}\right)
\end{array}\right]
$$

The kernel of this matrix is the vector $\mathbf{1}=[1,1, \ldots, 1]^{\top}$, which implies that a solution exists if and only if the sum of the components of the right-hand data vanishes. This may be easily verified thanks to the Galerkin property (see [3]).

Since the system (17) is singular the least square solution is selected. As a consequence, there exists a constant $C$, depending only on the number of elements in the patch $\widetilde{x}_{n}$ surrounding a vertex $x_{n}$, such that (for proof see for ex. [15])

$$
\sum_{K \in \widetilde{x}_{n}} \sigma_{K, n}^{2} \preceq \sum_{K \in \widetilde{x}_{n}} \widetilde{\Delta}_{K}\left(\theta_{n}\right)^{2}
$$

\subsection{Minimum energy extensions}

Minimum energy extensions were first introduced in the work of Ainsworth and Babuška [1]. These extensions play a key role in the construction of an estimator which is stable with respect to the perturbation parameter $\kappa$. The original equilibrated residual method is described in the work of Ainsworth and Oden [2]. However, as it is shown in [1], it is not stable with respect to $\kappa$. Ainsworth and Babuška [1] propose the following modification of the previous method for the singularly perturbed case. The functions $\theta_{n}$ in (17) are replaced by an approximate 

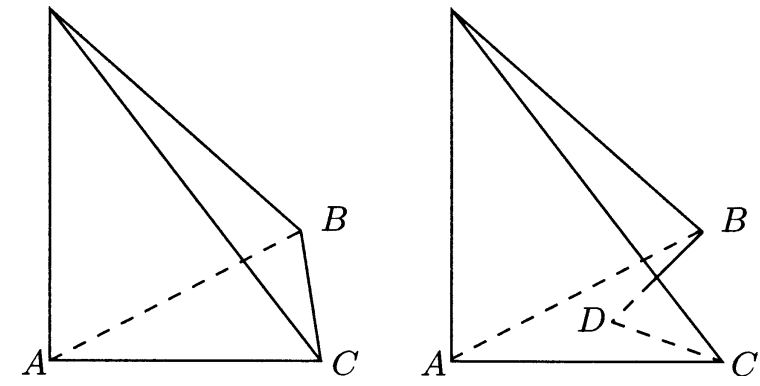

FIGURE 2. Original hat function and the family of functions used to approximate the minimum energy extension $\mathcal{E} \theta$.

minimum energy extension $\theta_{n}^{*}$ to $\left.\theta_{n}\right|_{\partial K}$. The system (17) then is solved in a least-square sense, since it has no solution in general. As in (20), one gets the solution that depends continuously on the data:

$$
\sum_{K \in \widetilde{x}_{n}} \sigma_{K, n}^{2} \preceq \sum_{K \in \widetilde{x}_{n}} \widetilde{\Delta}_{K}\left(\theta_{n}^{*}\right)^{2}
$$

The error estimator we propose is derived from the estimator of the work [1] but differs in two details. Firstly, we pay more attention to the minimization of the appropriate function energy norm and even obtain the minimum. We will develop this in this section. The second modification is described in Section 6 .

Let $K$ be any element and let $v \in H^{1 / 2}(\partial K)$. The minimum energy extension $\mathcal{E} v$ of $v$ to the interior of the element is characterized by the conditions

$$
\mathcal{E} v \in H^{1}(K): \mathcal{E} v=v \text { on } \partial K, \quad B_{K}(\mathcal{E} v, \omega)=0 \forall \omega \in H_{0}^{1}(K) .
$$

The definition of the minimum energy extension has an advantageous property. Let $v \in H^{1 / 2}(\partial K)$. The minimum energy extension $\mathcal{E} v$ of $v$ to the interior of the element has the minimal energy norm among all functions coinciding with $v$ on the boundary $\partial K$. Indeed, consider the energy norm of the function $\mathcal{E} v+\omega$ :

$$
\|\mathcal{E} v+\omega\|^{2}=\left\|\left|\mathcal{E} v\left\|^{2}+\right\|\right| \omega\left|\left\|^{2}+2 B_{K}(\mathcal{E} v, \omega)=\right\| \mathcal{E} v\right|\right\|^{2}+\left.\|\omega\|\right|^{2} \geq\left.\|\mathcal{E} v\|\right|^{2} .
$$

The proof easily follows from $(22)$ observing that $\mathcal{E} v+\omega$ coincides with $\mathcal{E} v$ on the boundary $\partial K$.

For the one-dimensional case it is possible to find a minimum energy extension explicitly (see [1]).

Consider now the two-dimensional case. We look for an approximation for the minimum energy extension of the first-order basis function. Let the element $K=\triangle A B C$ be a triangle. Consider the basis function $\theta$ corresponding to the vertex $A$. We seek an approximation to the minimum energy extension $\mathcal{E} \theta$ in the following class $\Lambda$ of functions. Set

$$
\begin{aligned}
\Lambda:= & \left\{v \in C^{0}(K): v=\theta \text { on } \partial K, v=0 \text { in } \triangle C D B,\right. \\
& v \text { is linear in each triangle } \triangle C A D \text { and } \triangle B A D, D \in \triangle A B C\} .
\end{aligned}
$$

We obtain now an approximation for the minimum energy extension of this basis function. To this end we put an arbitrary point $D$ in the triangle (see Fig. 2).

Next we choose that function from the set $\Lambda$ that minimizes energy norm. Our developments here differs from the original paper [1]. There a point $(1 / \kappa, 1 / \kappa)$ is introduced in the reference triangle and $D$ is the image of this point after the corresponding affine transformation. The corresponding function does not necessarily minimize the energy over $\Lambda$ but it is shown to be sufficiently accurate. For us, however this is not sufficient and we instead consider the point $D$ to be in the actual triangle in order to obtain the optimal position of this point. 


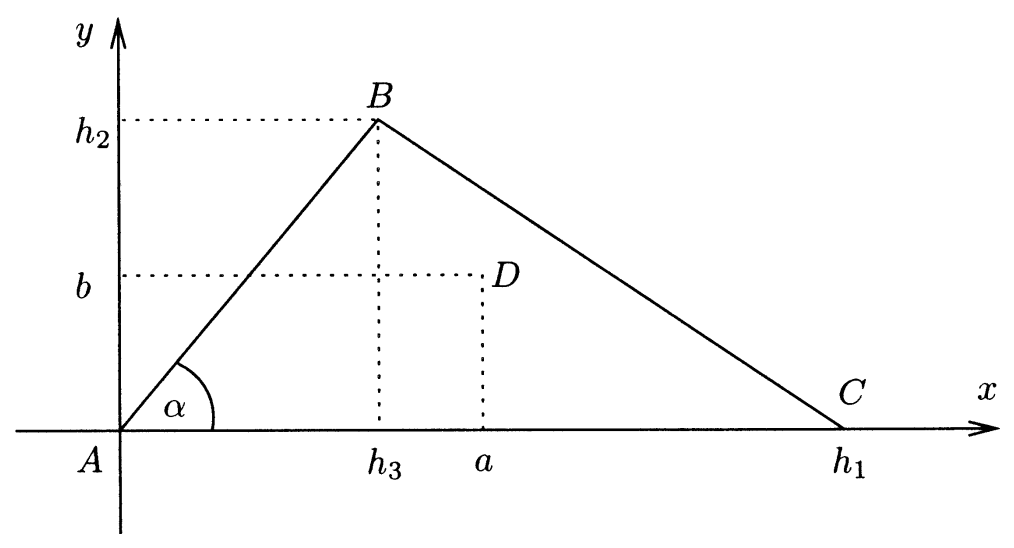

FIGURE 3. Notations for the parameters of an arbitrary triangle.

Introduce a local coordinate system such that the vertex $A$ coincides with the origin and the edge $A C$ lies on the axis $O x$. Let $D=(a, b), C=\left(h_{1}, 0\right)$ and $B=\left(h_{3}, h_{2}\right)$ (see Fig. 3).

Let $\Theta(a, b, x, y) \in \Lambda$ be an admissible function, see Figure 2. The squared energy norm of this function is

$$
\begin{aligned}
\Phi(a, b)= & B_{K}(\Theta(a, b, x, y), \Theta(a, b, x, y)) \\
= & \frac{\kappa^{2}}{12}\left(b\left(h_{1}-h_{3}\right)+a h_{2}\right)+\frac{2 h_{1} h_{2} h_{3}-a h_{1} h_{2}-b h_{1} h_{3}+b h_{3}^{2}}{2 h_{1} h_{3}^{2}} \\
& +\frac{\left(h_{2}^{2}+h_{3}^{2}\right)\left(a-h_{3}\right)^{2}}{2 h_{3}^{2}\left(a h_{2}-b h_{3}\right)}+\frac{\left(a-h_{1}\right)^{2}}{2 h_{1} b} .
\end{aligned}
$$

For given parameters $h_{1}, h_{2}, h_{3}$ we want to minimize $\Phi(a, b)$ with respect to $a$ and $b$. A number of calculations leads to a stationary point of this function

$$
\left\{\begin{aligned}
\bar{a}^{*} & =\frac{\sqrt{6 h_{1}}\left(h_{3}+\sqrt{h_{2}^{2}+h_{3}^{2}}\right)}{\sqrt{12 h_{3}+12 \sqrt{h_{2}^{2}+h_{3}^{2}}+\kappa^{2} h_{2}^{2} h_{1}}} \\
\bar{b}^{*} & =\left(-\frac{h_{3}}{h_{2}}+\sqrt{\left(\frac{h_{3}}{h_{2}}\right)^{2}+1}\right) \bar{a}^{*} .
\end{aligned}\right.
$$

To prove that this solution is a minimum point it is sufficient to show convexity of the function. Therefore consider the Hessian matrix $D^{2} \Phi$ of the second-order derivatives. By direct computations one obtains

$$
\begin{aligned}
\frac{\partial^{2} \Phi}{\partial a^{2}} & =\quad \frac{1}{b h_{1}}+\frac{\left(h_{2}^{2}+h_{3}^{2}\right)\left(b-h_{2}\right)^{2}}{\left(h_{2} a-h_{3} b\right)^{3}} \geq 0 \\
\operatorname{det} D^{2} \Phi & =\frac{\left(h_{2}^{2}+h_{3}^{2}\right)\left(h_{1} b-h_{3} b+h_{2} a-h_{1} h_{2}\right)^{2}}{\left(h_{2} a-h_{3} b\right)^{3} b^{3} h_{1}} \geq 0 .
\end{aligned}
$$

Hence $\Phi(a, b)$ is convex and $\left(\bar{a}^{*}, \bar{b}^{*}\right)$ is the unique minimum.

We have found the function $\Theta^{*}\left(\bar{a}^{*}, \bar{b}^{*}, x, y\right)$ which minimizes the energy norm over the set $\Lambda$. However, in practice it is sufficient to take not the exact values of $\bar{a}^{*}$ and $\bar{b}^{*}$, but some values $a^{*}, b^{*}$ that are equivalent for 


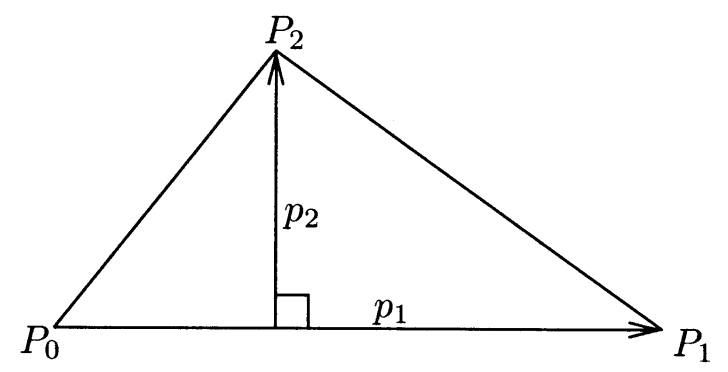

FiguRE 4. Notation of a triangle $K$.

$\kappa \rightarrow \infty$, namely

$$
\left\{\begin{array}{l}
a^{*}=\frac{\sqrt{6}}{\kappa} \frac{\left(h_{3}+\sqrt{h_{2}^{2}+h_{3}^{2}}\right)}{h_{2}} \\
b^{*}=\frac{\sqrt{6}}{\kappa} .
\end{array}\right.
$$

Note that the corresponding point $D=\left(a^{*}, b^{*}\right)$ lies on the bisector of the angle $\angle B A C=: \alpha$ and $|A D|=\frac{\sqrt{6}}{\kappa \sin \alpha / 2}$. The analysis given neglects the fact that $(a, b)$ should be contained in $K$. Therefore, we construct the function $\theta^{*}$ as follows:

$$
\theta^{*}:= \begin{cases}\Theta\left(a^{*}, b^{*}, x, y\right), & \text { if }\left(a^{*}, b^{*}\right) \in K, \\ \theta, & \text { otherwise. }\end{cases}
$$

Lemma 3.2. Under the above notations and assumptions the following holds

$$
\left\|\theta^{*}\right\|_{L_{2}(K)}^{2} \preceq|K| \min \left(1, h_{\min , K}^{-1} \kappa^{-1}\right) \sim \operatorname{meas}(\partial K) \min \left(h_{\min , K}, \kappa^{-1}\right),
$$

where $h_{\min , K}$ is the height corresponding to the largest edge of the triangle $K$.

Proof. Consider $K$ for which $\left(a^{*}, b^{*}\right) \in K$. A short calculation yields $\kappa^{-1} \preceq h_{\min , K}$ and $\min \left(h_{\min , K}, \kappa^{-1}\right) \sim \kappa^{-1}$. Furthermore one obtains

$$
\begin{aligned}
\left\|\theta^{*}\right\|_{L_{2}(K)}^{2} & =\frac{\sqrt{6}\left(h_{1}+\sqrt{h_{2}^{2}+h_{3}^{2}}\right)}{12 \kappa} \\
& \preceq \operatorname{meas}(\partial K) \kappa^{-1} \sim \operatorname{meas}(\partial K) \min \left(h_{\min , K}, \kappa^{-1}\right) .
\end{aligned}
$$

It remains to consider the case $\kappa^{-1} \gg h_{\min , K}$. In this case we have $\min \left(1, h_{\min , K^{-1}}^{-1}\right)=1$ and $\theta^{*}$ coincides with $\theta$. The estimate

completes the proof.

$$
\left\|\theta^{*}\right\|_{L_{2}(K)}^{2}=\|\theta\|_{L_{2}(K)}^{2} \sim|K|=|K| \min \left(1, h_{\min , K}^{-1} \kappa^{-1}\right)
$$

\section{Theoretical BACKGRound OF THE EQUILIBRATED RESIDUAL METHOD IN ANISOTROPIC CASE}

\subsection{Notation}

Let a triangulation $\mathcal{T}$ be given which satisfies the usual conformity condition (see [12], Chap. 2). Following the notation of Kunert [17], the three vertices of an arbitrary triangle $K \in \mathcal{T}$ are denoted by $P_{0}, P_{1}, P_{2}$ such that $P_{0} P_{1}$ is the longest edge of $K$. Additionally define two orthogonal vectors $p_{i}$ with lengths $h_{i, K}:=\left|p_{i}\right|$, see Figure 4. Observe that $h_{1, K}>h_{2, K}$ and set $h_{\min , K}:=h_{2, K}, h_{\max , K}:=h_{1, K}$. 
In addition to the usual conformity conditions of the mesh we assume that the following two properties hold.

1. The number of triangles containing a node $x_{n}$ is bounded uniformly.

2. The dimensions of adjacent triangles must not change rapidly, i.e.

$$
h_{i, K^{\prime}} \sim h_{i, K} \quad \forall K, K^{\prime} \text { with } \bar{K} \cap \overline{K^{\prime}} \neq \emptyset, i=1,2 .
$$

Define the matrices $A_{K}$ and $C_{K} \in \mathbb{R}^{2 \times 2}$ by

$$
A_{K}:=\left(\overrightarrow{P_{0} P_{1}}, \overrightarrow{P_{0} P_{2}}\right) \quad \text { and } \quad C_{K}:=\left(p_{1}, p_{2}\right)
$$

and introduce an affine linear mapping

$$
F_{A}(\mathbf{x}):=A_{K} \cdot \mathbf{x}+\vec{P}_{0}, \mathbf{x} \in \mathbb{R}^{2} .
$$

We will use the notion of the reference triangle $\hat{K}=F_{A}^{-1}(K)$ which is uniquely defined by the mapping $F_{A}$.

The following mesh dependent energy scalar product and norm will play a crucial role in the modification done in Section 6 to guarantee the robustness of the equilibrated residual method on anisotropic meshes.

Definition 4.1 (Mesh dependent energy scalar product). Let $K \in \mathcal{T}$ be any triangle, $u \in H_{0}^{1}(\Omega)$ and $v \in H^{1}(K)$, then we define the mesh-dependent energy scalar product and norms by

$$
\begin{aligned}
{ }_{m} B_{K}(u, v) & :=h_{\min , K}^{-2}\left(C_{K}^{\top} \nabla u, C_{K}^{\top} \nabla v\right)_{K}+\kappa^{2}(u, v)_{K}, \\
m\|u\|_{K} & :={ }_{m} B_{K}(u, u)^{1 / 2} \\
m\|u\| \| & :=\left(\sum_{K \in \mathcal{T}} m\|u\|_{K}^{2}\right)^{1 / 2} .
\end{aligned}
$$

The local mesh-dependent energy norm satisfies the following property

$$
\|\| u\|\|_{K} \leq{ }_{m}\|u \mid\|_{K} \leq \frac{h_{\max , K}}{h_{\min , K}}\|u\| \|_{K} .
$$

Note that the standard and mesh dependent energy norms are equivalent in the case of isotropic elements.

\subsection{Some basic inequalities}

This paragraph provides some facts which will be useful in the subsequent analysis for obtaining the lower error bound. The reader who are not interested in details of the theory may omit this paragraph without any affecting the understanding. The following two lemmata are are extended versions of the corresponding results from [21].

Lemma 4.2. Assume that $\varphi_{K} \in \mathbb{P}^{0}(K)$ and $\varphi_{\gamma} \in \mathbb{P}^{0}(\gamma)$. Then

$$
\begin{aligned}
\left\|b_{K}^{1 / 2} \varphi_{K}\right\|_{L_{2}(K)} & \sim\left\|\varphi_{K}\right\|_{L_{2}(K)} \\
\left\|b_{K} \varphi_{K}\right\|_{L_{2}(K)} & \sim\left\|\varphi_{K}\right\|_{L_{2}(K)} \\
\left\|\nabla\left(b_{K} \varphi_{K}\right)\right\|_{L_{2}(K)} & \preceq h_{\min , K}^{-1}\left\|\varphi_{K}\right\|_{L_{2}(K)} \\
\left\|C_{K}^{\top} \nabla\left(b_{K} \varphi_{K}\right)\right\|_{L_{2}(K)} & \preceq\left\|\varphi_{K}\right\|_{L_{2}(K)} \\
\left\|b_{K} \varphi_{K}\right\|_{K} & \preceq \min \left(h_{\min , K}, \kappa^{-1}\right)^{-1}\left\|\varphi_{K}\right\|_{L_{2}(K)} \\
m\left\|b_{K} \varphi_{K}\right\|_{K} & \preceq \min \left(h_{\min , K}, \kappa^{-1}\right)^{-1}\left\|\varphi_{K}\right\|_{L_{2}(K)} .
\end{aligned}
$$




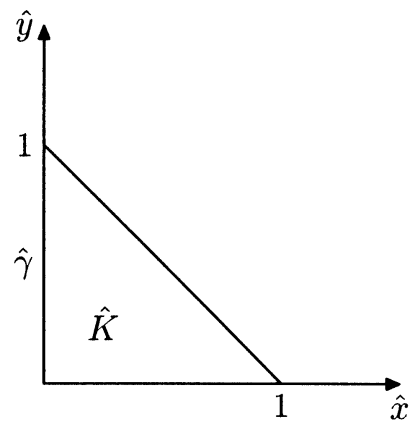

(a)

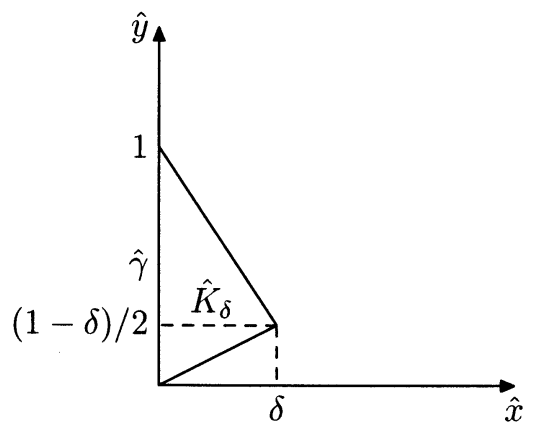

(b)

FiguRE 5. Definition of squeezed edge bubble functions: (a) - reference triangle $\hat{K}$, (b) - squeezed reference triangle $\hat{K}_{\delta}$.

Proof. Inequalities (23) and (25) are copied from [17] (p. 27, Lem. 2.7) without changes. Inequality (24) can be obtained analogously to (23). Inequality (26) is a refined version of (25) obtained by avoiding additional estimation on page 26, lines 3-4 from above in [17]. Another refined inequality (28) may be obtained in the same manner. Finally, combining (24) and (25) we show (27):

$\left\|b_{K} \varphi_{K}\right\|_{K}^{2}=\left\|\nabla\left(b_{K} \varphi_{K}\right)\right\|_{L_{2}(K)}^{2}+\kappa^{2}\left\|b_{K} \varphi_{K}\right\|_{L_{2}(K)}^{2} \preceq\left(h_{\min , K}^{-2}+\kappa^{2}\right)\left\|\varphi_{K}\right\|_{L_{2}(K)}^{2} \preceq \min \left(h_{\min , K}, \kappa^{-1}\right)^{-2}\left\|\varphi_{K}\right\|_{L_{2}(K)}^{2}$.

Again following [21], we define the squeezed edge bubble functions, extend the definition also for the squeezed spline functions and state the corresponding inverse inequalities. The definitions are given first for the reference triangle $\hat{K}$ and then for the actual triangle $K$.

Consider the reference triangle $\hat{K}$ and an edge $\hat{\gamma}$ thereof. Without loss of generality, assume that it lies on the axis $O \hat{y}$. By $\gamma$ we denote the corresponding edge on the boundary of actual triangle $K$. For a real number $\delta \in(0,1]$ define a linear mapping $F_{\delta}: \mathbb{R}^{2} \rightarrow \mathbb{R}^{2}$ by

$$
F_{\delta}(\mathbf{x}):=B_{\delta} \cdot \mathbf{x} \quad \text { with } B_{\delta}=\left(\begin{array}{cc}
\delta & 0 \\
\frac{1-\delta}{2} & 1
\end{array}\right) \in \mathbb{R}^{2 \times 2}
$$

Set $\hat{K}_{\delta}:=F_{\delta}(\hat{K})$, i.e. it is the triangle with the edge $\hat{\gamma}$ and a vertex at $\left(\delta, \frac{1-\delta}{2}\right)$, see Figure 5 .

Let $b_{\hat{\gamma}}$ be the usual edge bubble function of $\hat{\gamma}$ on $\hat{K}$. Define the squeezed bubble function $b_{\hat{\gamma}, \delta}$ by $b_{\hat{\gamma}, \delta}:=b_{\hat{\gamma}} \circ F_{\delta}^{-1}$, i.e. $b_{\hat{\gamma}, \delta}$ is the usual face bubble function of $\hat{\gamma}$ on the triangle $\hat{K}_{\delta}$. For clarity we recall that $b_{\hat{\gamma}, \delta}=0$ on $\hat{K} \backslash \hat{K}_{\delta}$.

Consider now an actual triangle $K$. The squeezed edge bubble function $b_{\gamma, \delta} \in H^{1}(K)$ of an edge $\gamma$ of $K$ is defined by $b_{\gamma, \delta}:=b_{\hat{\gamma}, \delta} \circ F_{A}^{-1}$. Analogously we can define the squeezed edge spline function $s_{\gamma, \delta}:=s_{\hat{\gamma}} \circ F_{\delta}^{-1} \circ F_{A}^{-1}$. The actual value of parameter $\delta$ will be specified later. The usual and squeezed edge bubble/spline functions are drawn in Figure 6.

We are interested in a particular value of parameter $\delta$ depending on the edge $\gamma$ for which the squeezed functions are defined. From now on we let

$$
\delta_{\gamma}:=\min \left(1, \kappa^{-1} h_{\min , \gamma}^{-1}\right)=h_{\min , \gamma}^{-1} \min \left(h_{\min , \gamma}, \kappa^{-1}\right)
$$

Lemma 4.3 (inverse inequalities for squeezed edge bubble/spline functions). Let $\gamma$ be an arbitrary face of $K$. Assume that $\varphi_{\gamma} \in \mathbb{P}^{0}(\gamma), \mu_{\gamma, \delta_{\gamma}} \in\left\{b_{\gamma, \delta_{\gamma}}, s_{\gamma, \delta_{\gamma}}\right\}$ and $\nu_{\gamma, \delta_{\gamma}} \in \operatorname{span}\left\{b_{\gamma, \delta_{\gamma}}, s_{\gamma, \delta_{\gamma}}\right\}$. Then the following inverse 


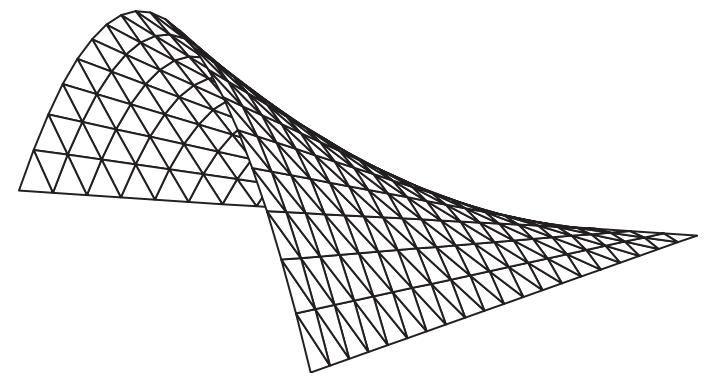

(a)

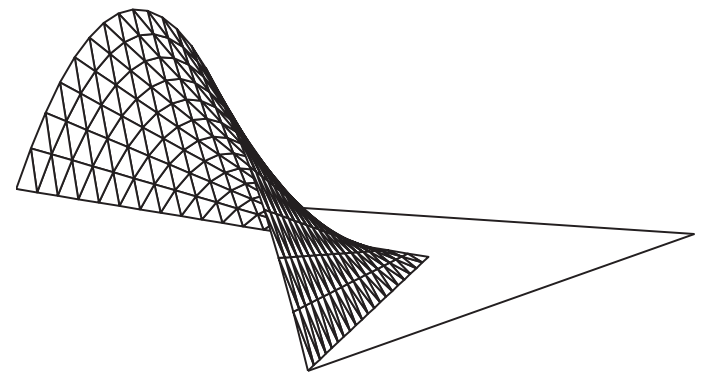

(c)

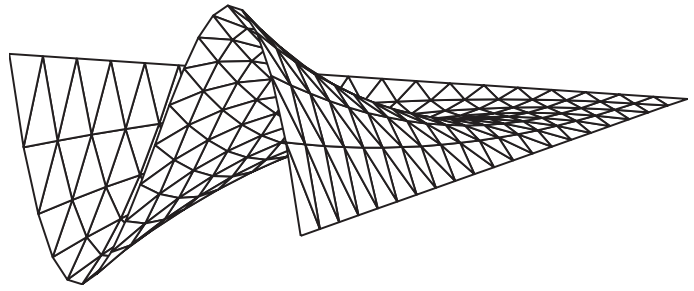

(b)

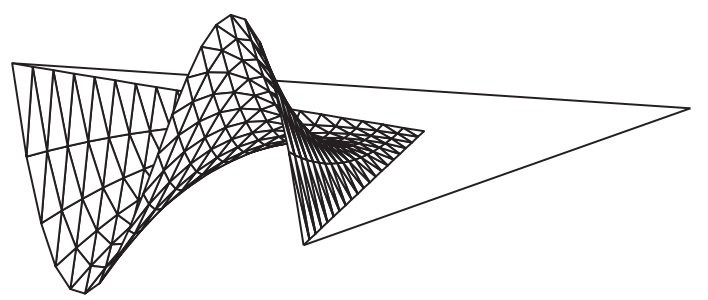

(d)

Figure 6. Bubble and spline functions: (a) - edge bubble function $b_{\gamma}$, (b) - edge spline function $s_{\gamma}$, (c) - squeezed edge bubble function $b_{\gamma, \delta},(\mathrm{d})$ - squeezed edge spline function $s_{\gamma, \delta}$.

inequalities hold:

$$
\begin{aligned}
& \left\|b_{\gamma, \delta_{\gamma}}^{1 / 2} \varphi_{\gamma}\right\|_{L_{2}(\gamma)} \sim\left\|\varphi_{\gamma}\right\|_{L_{2}(\gamma)} \\
& \left\|\mu_{\gamma, \delta_{\gamma}} \varphi_{\gamma}\right\|_{L_{2}(\gamma)} \sim\left\|\varphi_{\gamma}\right\|_{L_{2}(\gamma)} \\
& \left\|\mu_{\gamma, \delta_{\gamma}} F_{e x t}\left(\varphi_{\gamma}\right)\right\|_{L_{2}(K)} \preceq\left(\frac{|K|}{|\gamma|}\right)^{1 / 2} h_{\min , K}^{-1 / 2} \min \left(h_{\min , K}, \kappa^{-1}\right)^{1 / 2}\left\|\varphi_{\gamma}\right\|_{L_{2}(\gamma)} \\
& \left\|\nabla\left(\mu_{\gamma, \delta_{\gamma}} F_{\text {ext }}\left(\varphi_{\gamma}\right)\right)\right\|_{L_{2}(K)} \preceq\left(\frac{|K|}{|\gamma|}\right)^{1 / 2} h_{\min , K}^{-1 / 2} \min \left(h_{\min , K}, \kappa^{-1}\right)^{-1 / 2}\left\|\varphi_{\gamma}\right\|_{L_{2}(\gamma)} \\
& h_{\min , K}^{-1}\left\|C_{K}^{\top} \nabla\left(\mu_{\gamma, \delta_{\gamma}} F_{e x t}\left(\varphi_{\gamma}\right)\right)\right\|_{L_{2}(K)} \preceq\left(\frac{|K|}{|\gamma|}\right)^{1 / 2} h_{\min , K}^{-1 / 2} \min \left(h_{\min , K}, \kappa^{-1}\right)^{-1 / 2}\left\|\varphi_{\gamma}\right\|_{L_{2}(\gamma)} \\
& \left\|\mu_{\gamma, \delta_{\gamma}} F_{\text {ext }}\left(\varphi_{\gamma}\right)\right\|_{K} \preceq\left(\frac{|K|}{|\gamma|}\right)^{1 / 2} h_{\min , K}^{-1 / 2} \min \left(h_{\min , K}, \kappa^{-1}\right)^{-1 / 2}\left\|\varphi_{\gamma}\right\|_{L_{2}(\gamma)} \\
& { }_{m}\left\|\mu_{\gamma, \delta_{\gamma}} F_{\text {ext }}\left(\varphi_{\gamma}\right)\right\|_{K} \preceq\left(\frac{|K|}{|\gamma|}\right)^{1 / 2} h_{\min , K}^{-1 / 2} \min \left(h_{\min , K}, \kappa^{-1}\right)^{-1 / 2}\left\|\varphi_{\gamma}\right\|_{L_{2}(\gamma)} \\
& \left\|\nu_{\gamma, \delta_{\gamma}}\right\|_{L_{2}(K)} \preceq\left(\frac{|K|}{|\gamma|}\right)^{1 / 2} h_{\min , K}^{-1 / 2} \min \left(h_{\min , K}, \kappa^{-1}\right)^{1 / 2}\left\|\nu_{\gamma, \delta_{\gamma}}\right\|_{L_{2}(\gamma)} \\
& { }_{m}\left\|\nu_{\gamma, \delta_{\gamma}}\right\|\left\|_{K} \preceq\left(\frac{|K|}{|\gamma|}\right)^{1 / 2} h_{\min , K}^{-1 / 2} \min \left(h_{\min , K}, \kappa^{-1}\right)^{-1 / 2}\right\| \nu_{\gamma, \delta_{\gamma}} \|_{L_{2}(\gamma)} .
\end{aligned}
$$


Proof. We observe first that (29) is identical to (2.26) in [17] (p. 27, Lem. 2.7) and (30) can be obtained analogously. Inequalities (31) and (32) follow directly from the corresponding inequalities in [21] (p. 247, Lem. 3.7) extended also to the spline functions $s_{\gamma, \delta_{\gamma}}$. Inequality (34) follows from (31) and (32) in the similar way as (27) in Lemma 4.2. The proof of refined estimates (33) and (35) follows the lines of the corresponding proof of (26). In order to show (36) we express $\nu_{\gamma, \delta_{\gamma}}$ in the form $\nu_{\gamma, \delta_{\gamma}}=C_{b} b_{\gamma, \delta_{\gamma}}+C_{s} s_{\gamma, \delta_{\gamma}}$, where $C_{b}$ and $C_{s}$ are two constants, and show (36) utilizing the triangle inequality (31), and (30) subsequently:

$$
\begin{aligned}
\left\|\nu_{\gamma, \delta_{\gamma}}\right\|_{L_{2}(K)}^{2} & =\left\|C_{b} b_{\gamma, \delta_{\gamma}}+C_{s} s_{\gamma, \delta_{\gamma}}\right\|_{L_{2}(K)}^{2} \leq\left\|C_{b} b_{\gamma, \delta_{\gamma}}\right\|_{L_{2}(K)}^{2}+\left\|C_{s} s_{\gamma, \delta_{\gamma}}\right\|_{L_{2}(K)}^{2} \\
& \preceq \frac{|K|}{|\gamma|} h_{\min , K}^{-1} \min \left(h_{\min , K}, \kappa^{-1}\right)\left(\left\|C_{b}\right\|_{L_{2}(\gamma)}^{2}+\left\|C_{s}\right\|_{L_{2}(\gamma)}^{2}\right) \\
& \sim \frac{|K|}{|\gamma|} h_{\min , K}^{-1} \min \left(h_{\min , K}, \kappa^{-1}\right)\left(\left\|C_{b} b_{\gamma, \delta_{\gamma}}\right\|_{L_{2}(\gamma)}^{2}+\left\|C_{s} s_{\gamma, \delta_{\gamma}}\right\|_{L_{2}(\gamma)}^{2}\right) \\
& =\frac{|K|}{|\gamma|} h_{\min , K}^{-1} \min \left(h_{\min , K}, \kappa^{-1}\right)\left\|C_{b} b_{\gamma, \delta_{\gamma}}+C_{s} s_{\gamma, \delta_{\gamma}}\right\|_{L_{2}(\gamma)}^{2},
\end{aligned}
$$

where we used the orthogonality of functions $b_{\gamma, \delta_{\gamma}}$ and $s_{\gamma, \delta_{\gamma}}$ over edge $\gamma$. Estimate (37) may be obtained analogously.

From a heuristic point of view one should stretch the triangle in that direction where the (directional) derivative of the function shows little change. The better the anisotropic mesh $\mathcal{T}$ is aligned with the anisotropic function $v$, the more accurate one would expect the error estimates to be. In order to measure the alignment of $\mathcal{T}$ with $v$, Kunert $[17,18]$ has introduced the alignment measure $m_{1}(v, \mathcal{T})$ which is defined as follows.

Definition 4.4 (alignment measure $m_{1}$ ). Let $v \in H^{1}(\Omega)$ be an arbitrary non-constant function, and $\mathcal{F}$ be a family of triangulations of $\Omega$. Define the matching function $m_{1}(\cdot, \cdot): H^{1}(\Omega) \times \mathcal{F} \rightarrow \mathbb{R}$ by

$$
m_{1}(v, \mathcal{T}):=\frac{\left(\sum_{K \in \mathcal{T}} h_{\min , K}^{-2} \cdot\left\|C_{K}^{\top} \nabla v\right\|_{L_{2}(K)}^{2}\right)^{1 / 2}}{\|\nabla v\|} .
$$

Furthermore the local matching function $m_{1}(\cdot, \cdot): H^{1}(\Omega) \times \mathcal{T} \rightarrow \mathbb{R}$ is obviously defined by

$$
m_{1}(v, K):=h_{\min , K}^{-1} \frac{\left\|C_{K}^{\top} \nabla v\right\|_{L_{2}(K)}}{\|\nabla v\|_{L_{2}(K)}} .
$$

The alignment measure satisfies the following property:

$$
1 \leq m_{1}(v, \mathcal{T}) \leq C \max _{K \in \mathcal{T}} \frac{h_{\max , K}}{h_{\min , K}}
$$

The definition implies that a mesh $\mathcal{T}$ which is well aligned with an anisotropic function $v$, results in a small alignment measure $m_{1}(v, \mathcal{T})$.

Further we will need the anisotropic trace inequality and some more facts concerning approximation properties on an anisotropic triangle.

Lemma 4.5 (anisotropic trace inequality). Let $K$ be an arbitrary triangle and $\gamma$ be an edge of it. For $v \in H^{1}(K)$ the following trace inequality holds:

$$
\|v\|_{L_{2}(\gamma)}^{2} \preceq \frac{|\gamma|}{|K|}\|v\|_{L_{2}(K)}\left(\|v\|_{L_{2}(K)}+\left\|C_{K}^{\top} \nabla v\right\|_{L_{2}(K)}\right) .
$$


Lemma 4.6 (anisotropic approximation properties). Let $K$ be any triangle, $\gamma \subset \partial K$ be any edge thereof and $v \in H^{1}(K)$. Denote by $\bar{v}=\frac{1}{|K|} \int_{K} v$ the mean value of $v$ over an element $K$. Then

$$
\begin{aligned}
\|v-\bar{v}\|_{L_{2}(K)} & \leq\|v\|_{L_{2}(K)}, \\
\|v-\bar{v}\|_{L_{2}(K)} & \preceq\left\|C_{K}^{\top} \nabla v\right\|_{L_{2}(K)}, \\
\|v-\bar{v}\|_{L_{2}(K)} & \preceq \min \left(h_{\min , K}, \kappa^{-1}\right)_{m}\|v\|_{K} \preceq \min \left(h_{\min , K}, \kappa^{-1}\right) m_{1}(v, K)\|v\|_{K}, \\
\|v-\bar{v}\|_{L_{2}(\gamma)} & \preceq\left(\frac{|\gamma|}{|K|}\right)^{1 / 2}\left\|C_{K}^{\top} \nabla v\right\|_{L_{2}(K)}, \\
\|v-\bar{v}\|_{L_{2}(\gamma)} & \preceq\left(\frac{|\gamma|}{|K|}\right)^{1 / 2} h_{\min , K}^{1 / 2} \min \left(h_{\min , K}, \kappa^{-1}\right)^{1 / 2}{ }_{m}\|v\|_{K} \\
& \preceq\left(\frac{|\gamma|}{|K|}\right)^{1 / 2} h_{\min , K}^{1 / 2} \min \left(h_{\min , K}, \kappa^{-1}\right)^{1 / 2} m_{1}(v, K)\|v\|_{K} .
\end{aligned}
$$

Proof. Estimate (39) is obvious. For estimate (40) see for instance [18]. Estimate (41) evidently follows from (39) and (40). Combining Lemma 4.5 and estimate (39) of the current lemma and observing that $\|v\|_{L_{2}(K)}\left\|C_{K}^{\top} \nabla v\right\|_{L_{2}(K)} \leq\|v\|_{L_{2}(K)}^{2}+\left\|C_{K}^{\top} \nabla v\right\|_{L_{2}(K)}^{2}$ we verify (42). In order to show (43) we use (42) to obtain

$$
\|v-\bar{v}\|_{L_{2}(\gamma)}^{2} \preceq \frac{|\gamma|}{|K|}\left\|C_{K}^{\top} \nabla v\right\|_{L_{2}(K)}^{2} \leq \frac{|\gamma|}{|K|} h_{\min , K m}^{2}\|v\|_{K}^{2} .
$$

Furthermore, with the aid of Lemma 4.5 and estimates (39), (40) we get the following:

$$
\|v-\bar{v}\|_{L_{2}(\gamma)}^{2} \preceq \frac{|\gamma|}{|K|} \kappa^{-1} \sqrt{\kappa^{2}\|v\|_{L_{2}(K)}^{2}} h_{\min , K} \sqrt{h_{\min , K}^{-2}\left\|C_{K}^{\top} \nabla v\right\|_{L_{2}(K)}^{2}} \leq \frac{|\gamma|}{|K|} h_{\min , K} \kappa^{-1}{ }_{m}\|v\| \|_{K}^{2} .
$$

Combining the two previous estimates we get the result claimed.

\subsection{Estimates for element and face residuals in the anisotropic case}

In this section we prove two lemmas which we will need later. Namely, we derive the upper bounds for interior and face residuals. The jump discontinuity in the approximation of the normal flux at an interelement boundary is defined by

$$
\left[\frac{\partial u_{X}}{\partial n}\right]:=n_{K} \cdot\left(\nabla u_{X}\right)_{K}+n_{K^{\prime}} \cdot\left(\nabla u_{X}\right)_{K^{\prime}}
$$

and the usual interior and boundary residuals $r$ and $R$ are given by

$$
r:=f+\Delta u_{X}-\kappa^{2} u_{X}
$$

and

$$
R:= \begin{cases}-\left[\frac{\partial u_{X}}{\partial n}\right] & \text { on } \partial K \cap \partial K^{\prime} \\ 0 & \text { on } \partial K \cap \partial \Omega .\end{cases}
$$

Lemma 4.7 (residuals estimates). Let $K \in \mathcal{T}$ and $\gamma$ be any interior edge. Then

$$
\begin{aligned}
\|r\|_{L_{2}(K)} & \preceq \min \left(h_{\min , K}, \kappa^{-1}\right)^{-1}\|e\|_{K}+\|r-\bar{r}\|_{L_{2}(K)}, \\
\|R\|_{L_{2}(\gamma)} & \preceq \sum_{K^{\prime} \in \omega_{\gamma}}\left(\frac{\left|K^{\prime}\right|}{|\gamma|}\right)^{1 / 2} h_{\min , K^{\prime}}^{-1 / 2} \min \left(h_{\min , K^{\prime}}, \kappa^{-1}\right)^{-1 / 2}\left(\|e\|_{K^{\prime}}+\min \left(h_{\min , K^{\prime}}, \kappa^{-1}\right)\|r-\bar{r}\|_{L_{2}\left(K^{\prime}\right)}\right) .
\end{aligned}
$$


Proof. Let $v \in H_{0}^{1}(\Omega)$. Integrating by parts on each element yields

$$
B(e, v)=\sum_{K \in \mathcal{T}} \int_{K} r v \mathrm{~d} x-\sum_{\gamma \in \partial \mathcal{T}} \int_{\gamma} R v \mathrm{~d} s
$$

where $\partial \mathcal{T}$ denotes the collection of interelement edges. Hence for any $v \in H_{0}^{1}(\Omega)$

$$
B(e, v)=\sum_{K \in \mathcal{T}} \int_{K} \bar{r} v \mathrm{~d} x-\sum_{\gamma \in \partial \mathcal{T}} \int_{\gamma} R v \mathrm{~d} s+\sum_{K \in \mathcal{T}} \int_{K}(r-\bar{r}) v \mathrm{~d} x .
$$

Now, choosing $v:=b_{K} \bar{r}$ in the previous equality gives

$$
\int_{K} b_{K} \bar{r}^{2} \mathrm{~d} x=B_{K}\left(e, b_{K} \bar{r}\right)-\int_{K}(r-\bar{r}) b_{K} \bar{r} \mathrm{~d} x .
$$

Using (23), with the aid of Cauchy-Schwarz inequality we obtain

$$
\|\bar{r}\|_{L_{2}(K)}^{2} \preceq \int_{K} b_{K} \bar{r}^{2} \mathrm{~d} x \leq\left\|e\left|\|\|_{K}\right|\right\| b_{K} \bar{r} \mid\left\|_{K}+\right\| r-\bar{r}\left\|_{L_{2}(K)}\right\| b_{K} \bar{r} \|_{L_{2}(K)} .
$$

Estimates (24), (27) together with the triangle inequality imply (44).

We now show (45). Let $\gamma \in \partial \mathcal{T}$. Suppose that $\gamma=\bar{K}_{1} \cap \bar{K}_{2}$. Then $\omega_{\gamma}=\operatorname{int}\left(\bar{K}_{1} \cup \bar{K}_{2}\right)$. Choosing $v:=F_{\text {ext }}(R) b_{\gamma, \delta_{\gamma}} \in H_{0}^{1}(\Omega)$ in (46) implies

$$
\int_{\gamma} b_{\gamma, \delta_{\gamma}} R^{2} \mathrm{~d} s=\sum_{K \subset \omega_{\gamma}} \int_{K} r F_{e x t}(R) b_{\gamma, \delta_{\gamma}} \mathrm{d} x-B_{\omega_{\gamma}}\left(e, F_{e x t}(R) b_{\gamma, \delta_{\gamma}}\right) .
$$

Furthermore, applying the Cauchy-Schwarz inequality and (29), one obtains

$$
\|R\|_{L_{2}(\gamma)}^{2} \preceq \int_{\gamma} b_{\gamma, \delta_{\gamma}} R^{2} \mathrm{~d} s \leq \sum_{K \subset \omega_{\gamma}}\|r\|_{L_{2}(K)}\left\|F_{e x t}(R) b_{\gamma, \delta_{\gamma}}\right\|_{L_{2}(K)}+\|e\|\left\|_{\omega_{\gamma}}\right\| F_{e x t}(R) b_{\gamma, \delta_{\gamma}} \|_{\omega_{\gamma}} .
$$

The desired inequality (45) follows now from (31),(34) and the first result of the current lemma (44).

Recall that we use the procedure for finding approximate fluxes described in Section 3.2 with the functions $\theta_{n}$ replaced by $\theta_{n}^{*}$ in the system (17). In the singularly perturbed case and using anisotropic elements we have the following theorem.

Theorem 4.8 (stability of the approximate fluxes). Suppose that the finite element subspace $X$ is constructed using first-order (linear) elements on a partition $\mathcal{T}$ of the domain $\Omega$ into triangular elements. Let $\left\{g_{K}\right\}$ be the set of approximate fluxes, produced by the algorithm described in Section 3.2 with the functions $\theta_{n}$ replaced by $\theta_{n}^{*}, n \in \mathcal{N}$. Then, for each edge $\gamma$ of any element $K$,

$$
\begin{aligned}
\left\|g_{K}-\left\langle\frac{\partial u_{X}}{\partial n_{K}}\right\rangle\right\|_{L_{2}(\gamma)} \preceq \sum_{K^{\prime} \subset \omega_{K}}\left(\frac{\left|K^{\prime}\right|}{|\gamma|}\right)^{1 / 2} h_{\min , K^{\prime}}^{-1 / 2} \min \left(h_{\min , K^{\prime}}, \kappa^{-1}\right)^{-1 / 2} \\
\times\left(\|e\|_{K^{\prime}}+\min \left(h_{\min , K^{\prime}}, \kappa^{-1}\right)\|r-\bar{r}\|_{L_{2}\left(K^{\prime}\right)}\right) .
\end{aligned}
$$

Proof. Let $K \in \mathcal{T}$ be a fixed element and $\gamma \subset K$ be an edge thereof. Then

$$
\left.\left(g_{K}-\left\langle\frac{\partial u_{X}}{\partial n_{K}}\right\rangle\right)\right|_{\gamma} \in \mathbb{P}_{1}(\gamma)
$$


Following Section 3.2 the moments of this quantity are

$$
\stackrel{*}{\mu}_{K, n}^{\gamma}=\int_{\gamma}\left(g_{K}-\left\langle\frac{\partial u_{X}}{\partial n_{K}}\right\rangle\right) \theta_{n} \mathrm{~d} s
$$

By analogy with (10),

$$
\left.\left(g_{K}-\left\langle\frac{\partial u_{X}}{\partial n_{K}}\right\rangle\right)\right|_{\gamma}=\stackrel{*}{\mu}_{K, l}^{\gamma} \psi_{l}+\stackrel{*}{\mu}_{K, r}^{\gamma} \psi_{r}
$$

Therefore,

$$
\left\|g_{K}-\left\langle\frac{\partial u_{X}}{\partial n_{K}}\right\rangle\right\|_{L_{2}(\gamma)} \leq\left|\stackrel{*}{\mu}_{K, l}^{\gamma}\right|\left\|\psi_{l}\right\|_{L_{2}(\gamma)}+\left|\stackrel{*}{\mu}_{K, r}\right|\left\|\psi_{r}\right\|_{L_{2}(\gamma)}
$$

and since

$$
\left\|\psi_{l}\right\|_{L_{2}(\gamma)}^{2}=\left\|\psi_{r}\right\|_{L_{2}(\gamma)}^{2}=C|\gamma|^{-1}
$$

it follows that

$$
\left\|g_{K}-\left\langle\frac{\partial u_{X}}{\partial n_{K}}\right\rangle\right\|_{L_{2}(\gamma)}^{2} \preceq|\gamma|^{-1} \sum_{n \in \mathcal{N}(\gamma)}\left|\mu_{K, n}^{*}\right|^{2} .
$$

With the aid of (19), we conclude that

$$
\int_{\gamma}\left\langle\frac{\partial u_{X}}{\partial n_{K}}\right\rangle \theta_{n} \mathrm{~d} s= \begin{cases}\frac{1}{2}\left(\widetilde{\mu}_{K, n}^{\gamma}-\widetilde{\mu}_{K^{\prime}, n}^{\gamma}\right) & \text { on } \gamma=\partial K \cap \partial K^{\prime} \\ \widetilde{\mu}_{K, n}^{\gamma} & \text { on } \gamma=\partial K \cap \partial \Omega\end{cases}
$$

with $\widetilde{\mu}_{K, n}^{\gamma}$ defined in (13). Hence, thanks to (16),

$$
\stackrel{*}{\mu}_{K, n}^{\gamma}= \begin{cases}\frac{1}{2}\left(\sigma_{K, n}-\sigma_{K^{\prime}, n}\right) & \text { on } \gamma=\partial K \cap \partial K^{\prime} \\ \sigma_{K, n} & \text { on } \gamma=\partial K \cap \partial \Omega\end{cases}
$$

where the unknowns $\left\{\sigma_{K, n}\right\}$ are determined from conditions (17) and satisfy (20). It follows that

$$
\left|\mu_{K, n}^{* \gamma}\right|^{2} \preceq \sum_{K^{\prime} \in \widetilde{x}_{n}} \sigma_{K^{\prime}, n}^{2} \preceq \sum_{K^{\prime} \in \widetilde{x}_{n}} \widetilde{\Delta}_{K^{\prime}}\left(\theta_{n}^{*}\right)^{2} .
$$

The terms appearing on the right-hand side may be bounded by first recalling (18),

$$
\widetilde{\Delta}_{K^{\prime}}\left(\theta_{n}^{*}\right)=B_{K^{\prime}}\left(u_{X}, \theta_{n}^{*}\right)-\left(f, \theta_{n}^{*}\right)_{K^{\prime}}-\int_{\partial K^{\prime}}\left\langle\frac{\partial u_{X}}{\partial n_{K^{\prime}}}\right\rangle \theta_{n}^{*} \mathrm{~d} s
$$

then, integrating by parts reveals that

$$
\widetilde{\Delta}_{K^{\prime}}\left(\theta_{n}^{*}\right)=-\left(r, \theta_{n}^{*}\right)_{K^{\prime}}-\int_{\partial K^{\prime}} R \theta_{n}^{*} \mathrm{~d} s
$$


We proceed applying the Cauchy-Schwarz inequality and using Lemma 3.2 and Lemma 4.7:

$$
\begin{aligned}
& \left|\widetilde{\Delta}_{K^{\prime}}\left(\theta_{n}^{*}\right)\right| \leq\|r\|_{L_{2}\left(K^{\prime}\right)}\left\|\theta_{n}^{*}\right\|_{L_{2}\left(K^{\prime}\right)}+\sum_{\gamma^{\prime} \subset \partial K^{\prime} \cap \mathcal{E}_{n}}\|R\|_{L_{2}\left(\gamma^{\prime}\right)}\left\|\theta_{n}^{*}\right\|_{L_{2}\left(\gamma^{\prime}\right)} \\
& \preceq\left(\min \left(h_{\min , K^{\prime}}, \kappa^{-1}\right)^{-1} \mid\|e\|_{K^{\prime}}+\|r-\bar{r}\|_{L_{2}\left(K^{\prime}\right)}\right)\left|K^{\prime}\right|^{1 / 2} h_{\min , K^{\prime}}^{-1 / 2} \min \left(h_{\min , K^{\prime}}, \kappa^{-1}\right)^{1 / 2} \\
& +\sum_{\gamma^{\prime} \subset \partial K^{\prime} \cap \mathcal{E}_{n}} \sum_{K^{\prime \prime} \subset \omega_{\gamma^{\prime}}}\left|K^{\prime \prime}\right|^{1 / 2} h_{\min , K^{\prime \prime}}^{-1 / 2} \min \left(h_{\min , K^{\prime \prime}}, \kappa^{-1}\right)^{-1 / 2}\left(\|e\|_{K^{\prime \prime}}+\min \left(h_{\min , K^{\prime \prime}}, \kappa^{-1}\right)\|r-\bar{r}\|_{L_{2}\left(K^{\prime \prime}\right)}\right) \\
& \preceq \sum_{K^{\prime \prime} \in \widetilde{x}_{n}}\left|K^{\prime \prime}\right|^{1 / 2} h_{\min , K^{\prime \prime}}^{-1 / 2}\left(\min \left(h_{\min , K^{\prime \prime}}, \kappa^{-1}\right)^{-1 / 2}\|e\|_{K^{\prime \prime}}+\min \left(h_{\min , K^{\prime \prime}}, \kappa^{-1}\right)^{1 / 2}\|r-\bar{r}\|_{L_{2}\left(K^{\prime \prime}\right)}\right) .
\end{aligned}
$$

Hence,

$$
\sum_{K^{\prime} \in \widetilde{x}_{n}}\left|\widetilde{\Delta}_{K^{\prime}}\left(\theta_{n}^{*}\right)\right|^{2} \preceq \sum_{K^{\prime} \in \widetilde{x}_{n}}\left|K^{\prime}\right| h_{\min , K^{\prime}}^{-1}\left(\min \left(h_{\min , K^{\prime}}, \kappa^{-1}\right)^{-1}\|e\|_{K^{\prime}}^{2}+\min \left(h_{\min , K^{\prime}}, \kappa^{-1}\right)\|r-\bar{r}\|_{L_{2}\left(K^{\prime \prime}\right)}^{2}\right) .
$$

Combining (47), (48) and (49) leads to the result claimed.

\section{LOWER ERRor BOUND OF THE ORIGinal Ainsworth-BABUŠKa estimator IN THE ANISOTROPIC SINGULARLY PERTURBED CASE}

Describing in Section 3 the equilibrated residual method, we derived the upper error bound. The original analysis of the lower error bound for isotropic triangles dates back to the work by Ainsworth and Babuška [1]. Here we analyse the anisotropic case. It turns out that the original error estimator described in [1] has degenerating lower error bound.

The right hand side of the local problem (7) is originally defined as a linear functional only for the functions $v \in V_{K}$. We will need, however, to apply this functional also to the functions outside of $V_{K}$, namely to those not preserving the Dirichlet boundary conditions. To this end we introduce a new notation for the residual functional on the right hand side of (7):

$$
\mathcal{B}_{K}(v):=(f, v)_{K}-B_{K}\left(u_{X}, v\right)+\int_{\partial K} g_{K} v \mathrm{~d} s \quad \forall v \in H^{1}(K) .
$$

From this notation it is clear that $\mathcal{B}_{K}(v)=B_{K}\left(\phi_{K}, v\right)$ for all $v \in V_{K}$, but the domain of definition of the functional $\mathcal{B}_{K}$ is larger for the elements $K$ touching the boundary. The next lemma states some stability properties of the residual functional $\mathcal{B}_{K}$ which we will require in the lower error bound estimates.

Lemma 5.1. Let $\phi_{K}$ denote the solution of the local residual problem (7) for the error estimator on element $K$. Then, for any $v \in H^{1}(K)$,

$$
\left|\mathcal{B}_{K}(v-\bar{v})\right| \preceq m_{1}(v, K)\left(\|\mid\|\left\|_{\omega_{K}}+\min \left(h_{\min , K}, \kappa^{-1}\right)\right\| r-\bar{r} \|_{L_{2}\left(\omega_{K}\right)}\right)\|v \mid\|_{K} .
$$

Furthermore, if $\kappa \succeq h_{\min , K}^{-1}$, then

$$
\begin{aligned}
\left|\bar{\phi}_{K} \mathcal{B}_{K}(1)\right| & \preceq\left(\|\mid\|\left\|_{\omega_{K}}+\kappa^{-1}\right\| r-\bar{r} \|_{L_{2}\left(\omega_{K}\right)}\right)_{m}\left\|\phi_{K} \mid\right\|_{K} \\
& \leq m_{1}\left(\phi_{K}, K\right)\left(\left\|\left|\|\mid\|_{\omega_{K}}+\kappa^{-1}\|r-\bar{r}\|_{L_{2}\left(\omega_{K}\right)}\right)\right\| \phi_{K} \mid \|_{K} .\right.
\end{aligned}
$$

Proof. 1. Integrating (50) by parts yields

$$
\mathcal{B}_{K}(v-\bar{v})=\int_{K} r(v-\bar{v}) \mathrm{d} x+\frac{1}{2} \int_{\partial K} R(v-\bar{v}) \mathrm{d} s+\int_{\partial K}\left(g_{K}-\left\langle\frac{\partial u_{X}}{\partial n_{K}}\right\rangle\right)(v-\bar{v}) \mathrm{d} s .
$$


and it therefore follows that

$$
\begin{gathered}
\left|\mathcal{B}_{K}(v-\bar{v})\right| \leq \sum_{\gamma \subset \partial K}\left\|g_{K}-\left\langle\frac{\partial u_{X}}{\partial n_{K}}\right\rangle\right\|_{L_{2}(\gamma)}\|v-\bar{v}\|_{L_{2}(\gamma)} \\
+\|r\|_{L_{2}(K)}\|v-\bar{v}\|_{L_{2}(K)}+\frac{1}{2} \sum_{\gamma \subset \partial K}\|R\|_{L_{2}(\gamma)}\|v-\bar{v}\|_{L_{2}(\gamma)} .
\end{gathered}
$$

Combining results from Lemma 4.7, Theorem 4.8 and Lemma 4.6 we get (51).

2. Suppose that $\kappa \succeq h_{\min , K}^{-1}$. Then

$$
\mathcal{B}_{K}(1)=(f, 1)_{K}-B_{K}\left(u_{X}, 1\right)+\int_{\partial K} g_{K} \mathrm{~d} s .
$$

Integrating by parts, applying the Cauchy-Schwarz inequality, and estimating each term using Lemma 4.7 and Theorem 4.8 yield

$$
\begin{aligned}
\left|\mathcal{B}_{K}(1)\right| & \leq|K|^{1 / 2}\|r\|_{L_{2}(K)}+\frac{1}{2} \sum_{\gamma \in \partial K}|\gamma|^{1 / 2}\|R\|_{L_{2}(\gamma)}+\sum_{\gamma \in \partial K}|\gamma|^{1 / 2}\left\|g_{K}-\left\langle\frac{\partial u_{X}}{\partial n_{K}}\right\rangle\right\|_{L_{2}(\gamma)} \\
& \preceq \kappa|K|^{1 / 2}\left(\|\mid\|\left\|_{\omega_{K}}+\kappa^{-1}\right\| r-\bar{r} \|_{L_{2}\left(\omega_{K}\right)}\right),
\end{aligned}
$$

where the inequality $\min \left(h_{\min , K}, \kappa^{-1}\right) \preceq \kappa^{-1}$ has been used. Inequality (52) now can be easily obtained

$$
\begin{aligned}
\left|\bar{\phi}_{K} \mathcal{B}_{K}(1)\right| & \preceq \kappa|K|^{1 / 2}\left|\bar{\phi}_{K}\right|\left(\left|\|e \mid\|_{\omega_{K}}+\kappa^{-1}\|r-\bar{r}\|_{L_{2}\left(\omega_{K}\right)}\right)\right. \\
& \preceq \kappa\left\|\phi_{K}\right\|_{L_{2}(K)}\left(\|\mid\|\left\|_{\omega_{K}}+\kappa^{-1}\right\| r-\bar{r} \|_{L_{2}\left(\omega_{K}\right)}\right) \leq\left(\|\mid\|\left\|_{\omega_{K}}+\kappa^{-1}\right\| r-\bar{r} \|_{L_{2}\left(\omega_{K}\right)}\right)_{m}\|\|_{K}\|\|_{K}
\end{aligned}
$$

For the lower bound we have the following result.

Theorem 5.2 (Lower error bound). Let $g_{K}$ be the set of fluxes produced by the algorithm described in Section 3.2 with the functions $\theta_{n}$ replaced by $\theta_{n}^{*}$, and let $\phi_{K} \in V_{K}$ denote the solution of the local residual problem (7). Then,

$$
\left\|\phi_{K}\right\|_{K} \preceq m_{1}\left(\phi_{K}, K\right)\left(\|||\|_{\omega_{K}}+\min \left(h_{\min , K}, \kappa^{-1}\right)\|r-\bar{r}\|_{L_{2}\left(\omega_{K}\right)}\right) .
$$

If $\kappa$ vanishes, then $\min \left(h_{\min , K}, \kappa^{-1}\right)$ is replaced by $h_{\min , K}$.

Proof. Observe that for any $v \in V_{K}$,

$$
B_{K}\left(\phi_{K}, v\right)=\mathcal{B}_{K}(v)=\mathcal{B}_{K}(v-\bar{v})+\bar{v} \mathcal{B}_{K}(1)
$$

First, suppose $\kappa h_{\min , K} \succeq 1$ so that, in particular, $\kappa$ is positive and $\min \left(h_{\min , K}, \kappa^{-1}\right)^{-1} \sim \kappa$. Therefore, with the aid of Lemma 5.1,

$$
\left|\mathcal{B}_{K}\left(\phi_{K}-\bar{\phi}_{K}\right)\right| \preceq m_{1}\left(\phi_{K}, K\right)\left\{\|\mid\|\left\|_{\omega_{K}}+\min \left(h_{\min , K}, \kappa^{-1}\right)\right\| r-\bar{r} \|_{L_{2}\left(\omega_{K}\right)}\right\}\left|\left\|\phi_{K} \mid\right\|_{K}\right.
$$

Choosing $v$ to be equal to $\phi_{K}$ in (54), together with the above estimate, proves that the result holds for all elements $K$ satisfying $\kappa h_{\min , K} \succeq 1$.

The remaining elements satisfy $\kappa h_{\min , K} \ll 1$. Thanks to the assumptions on the partition, the condition $\kappa h_{K^{\prime}} \ll 1$ is satisfied by all elements $K^{\prime}$ contained in the patch $\omega_{K}$. Therefore, Lemma 3.2 reveals that the modified basis functions reduce to the standard basis functions on the patch. Consequently, the approximate fluxes will actually satisfy the equilibration conditions (9) exactly. Moreover, since

$$
\mathcal{B}_{K}(1)=(f, 1)_{K}-B_{K}\left(u_{X}, 1\right)+\int_{\partial K} g_{K} \mathrm{~d} s=0,
$$


the second term in (54) vanishes. The first estimate in Lemma 5.1 then completes the proof.

Theorem 5.2 gives the lower error bound of the true error. The main danger for reliability of the estimator is the function $m_{1}\left(\phi_{K}, K\right)$ presented on the right hand side. One cannot guarantee that the approximation for the error $\phi_{K}$ is aligned as well as the true error $e$. Unfortunately, it may happen so that the alignment of the approximation $\phi_{K}$ on the element $K$ is much worse then $e: m_{1}\left(\phi_{K}, K\right) \gg m_{1}(e, K)$. To avoid this problem a modification is proposed in the next paragraph.

\section{Modified EQUILIBRATED RESIDUAL METHOD}

For finding the equilibrated fluxes we use again the equilibrated residual method described in Section 3.2. In this paragraph, we propose an alternative method by changing the local problem, namely, instead of (7) we use

$$
{ }_{m} B_{K}\left(\phi_{K}, v\right)=(f, v)_{K}-B_{K}\left(u_{X}, v\right)+\int_{\partial K} g_{K} v \mathrm{~d} s \quad \forall v \in V_{K} .
$$

This local problem differs from the original local problem (7) only in the scalar product ${ }_{m} B_{K}(u, v)$ on the left hand side. The quantity $\phi_{K}$ is then not equivalent to the error $e$, but we will show that the ${ }_{m}\left\|\phi_{K}\right\| \|_{K}$ is related to $\|e\|_{K}$. The following two theorems give upper and lower bounds for the error.

Theorem 6.1 (reliability). Let $\left\{g_{K}: K \in \mathcal{T}\right\}$ be any set of boundary fluxes satisfying condition (5). In addition, if the absolute term $\kappa$ vanishes, then it is assumed that the fluxes satisfy the equilibration condition (8) on all elements that do not abut the boundary $\partial \Omega$. Then, the global error residual may be decomposed into local contributions

$$
B(e, v)=L(v)-B\left(u_{X}, v\right)=\sum_{K \in \mathcal{T}}{ }_{m} B_{K}\left(\phi_{K}, v\right), \quad v \in H^{1}(K),
$$

where $\phi_{K} \in V_{K}$ is the solution of the local problem (56). The global error in the finite element approximation may be bounded by

$$
\|e\|^{2} \leq m_{1}(e, \mathcal{T})^{2} \sum_{K \in \mathcal{T}} m\left\|\phi_{K}\right\|_{K}^{2},
$$

where $m_{1}(e, \mathcal{T})$ is the matching function introduced by $(38)$.

Proof. Using the representation of $B(e, v)$ in the local terms and subsequently applying the Cauchy-Schwarz inequality and the definition of the matching function, we have:

$$
\begin{aligned}
|B(e, v)| & =\left|\sum_{K \in \mathcal{T}}\left\{(f, v)_{K}-B_{K}\left(u_{X}, v\right)\right\}\right| \\
& =\left|\sum_{K \in \mathcal{T}}\left\{(f, v)_{K}-B_{K}\left(u_{X}, v\right)+\int_{\partial K} g_{K} v \mathrm{~d} s\right\}\right| \\
& =\left|\sum_{K \in \mathcal{T}} m B_{K}\left(\phi_{K}, v\right)\right| \\
& \leq \sum_{K \in \mathcal{T}} m\left\|\phi _ { K } \left|\left\|_{K} m \mid\right\| v \|_{K}\right.\right. \\
& \leq \sqrt{\sum_{K \in \mathcal{T}} m \mid\left\|\phi_{K}\right\| \|_{K}^{2}} \cdot \sqrt{\sum_{K \in \mathcal{T}}\left(h_{\min , K}^{-2}\left\|C_{K}^{\top} \nabla v\right\|_{L_{2}(K)}^{2}+\kappa^{2}\|v\|_{L_{2}(K)}^{2}\right)} \\
& \leq \sqrt{\sum_{K \in \mathcal{T}} m\left\|\phi_{K}\right\|_{K}^{2}} \cdot \sqrt{m_{1}(v, \mathcal{T})^{2}\|\nabla v\|_{L_{2}(\Omega)}^{2}+\kappa^{2}\|v\|_{L_{2}(\Omega)}^{2}} \\
& \leq m_{1}(v, \mathcal{T})\|v\| \| \sqrt{\sum_{K \in \mathcal{T}} m\left\|\phi_{K}\right\|_{K}^{2}} \cdot
\end{aligned}
$$

The substitution $v:=e$ completes the proof. 
Theorem 6.1 gives the usual result for anisotropic error estimators. See for instance [18,21].

Theorem 6.2 (efficiency). Let $g_{K}$ be the set of approximate fluxes produced by the algorithm described in Section 3.2 with the functions $\theta$ replaced by $\theta^{*}$, and let $\phi_{K} \in V_{K}$ denote the solution of the local residual problem (56). Then,

$$
{ }_{m}\left\|\phi _ { K } \left|\left\|_{K} \preceq \mid\right\| e\left\|_{\widetilde{K}}+\min \left(h_{\min , K}, \kappa^{-1}\right)\right\| r-\bar{r} \|_{L_{2}(\widetilde{K})} .\right.\right.
$$

Proof. The proof follows the same lines as the proof of the Theorem 5.2.

These two theorems are part of the main result of this work and guarantee the reliability and efficiency of the estimator, assuming an exact solution of the local problems.

\section{Computable approximation for the solution of the local Problem}

Up to this time we considered the infinite dimensional local problems (7) and (56). The author has not found any result in the literature saying that some computable approximation $\widetilde{\phi}_{K}$ is equivalent to $\phi_{K}$ in the energy norm,

$$
\left\|\widetilde{\phi}_{K}\left|\|\sim\| \phi_{K}\right|\right\|
$$

even not for isotropic elements. In the current section we construct an approximation $\widetilde{\phi}_{K}$ so that (57) holds. To this end we restrict the space $V_{K}$ to the space of bubbles/splines $V_{b}(K)$ defined in the following way:

$$
V_{b}(K):=\operatorname{span}\left\{b_{K}, b_{\gamma, \delta_{\gamma}}, s_{\gamma, \delta_{\gamma}}: \gamma \in \partial K \backslash \partial \Omega\right\} \subset V_{K}
$$

By means of the space $V_{b}(K)$ we can define the function $\widetilde{\phi}_{K} \in V_{b}(K)$ as the solution of the local finite dimensional problem

$$
{ }_{m} B_{K}\left(\widetilde{\phi}_{K}, v\right)=(f, v)_{K}-B_{K}\left(u_{X}, v\right)+\int_{\partial K} g_{K} v \mathrm{~d} s \quad \forall v \in V_{b}(K) .
$$

We prove the lower bound for $\phi_{K}$ first.

Theorem 7.1. Let $\phi_{K}$ and $\widetilde{\phi}_{K}$ be the solutions to the problems (56) and (58), respectively. Then

$$
m\left|\left\|\widetilde{\phi}_{K}\right\|_{K} \leq m\right|\left\|\phi_{K}\right\|_{K} .
$$

Proof. Substituting $v=\widetilde{\phi}_{K} \in V_{b}(K) \subset V_{K}$ in (58) and subsequently utilizing the Cauchy-Schwarz inequality we estimate ${ }_{m} B_{K}\left(\widetilde{\phi}_{K}, \widetilde{\phi}_{K}\right)$ as follows:

$$
\begin{aligned}
{ }_{m} B_{K}\left(\widetilde{\phi}_{K}, \widetilde{\phi}_{K}\right) & =\left(f, \widetilde{\phi}_{K}\right)_{K}-B_{K}\left(u_{X}, \widetilde{\phi}_{K}\right)+\int_{\partial K} g_{K} \widetilde{\phi}_{K} \mathrm{~d} s \\
& ={ }_{m} B_{K}\left(\phi_{K}, \widetilde{\phi}_{K}\right) \leq{ }_{m}\left\|\phi_{K}\right\|\left\|_{K m}\right\| \widetilde{\phi}_{K}\|\|_{K} .
\end{aligned}
$$

Dividing both sides by $m\left|\left\|\widetilde{\phi}_{K} \mid\right\|_{K}\right.$ we get the result claimed.

For further investigations we need the following preliminary result.

Lemma 7.2. Let $\phi_{K}$ denote the solution to the local residual problem (56) for the error estimator on the element $K$. If $\kappa \succeq h_{\min , K}^{-1}$, then

$$
\left|\bar{\phi}_{K} \mathcal{B}_{K}(1)\right| \preceq\left(\kappa^{-1}\|r\|_{L_{2}(K)}+\sum_{\gamma \subset \partial K} \kappa^{-1}\left(\frac{|\gamma|}{|K|}\right)^{1 / 2}\left\|g_{K}-\frac{\partial u_{x}}{\partial n_{K}}\right\|_{L_{2}(\gamma)}\right){ }_{m}\left\|\phi_{K} \mid\right\|_{K},
$$

where $\mathcal{B}_{K}$ is the residual functional defined in (50). 
Proof. The proof is done analogously to the proof of (52) in Lemma 5.1.

For the upper bound we employ the technique that is usually used in obtaining the lower error bound in the residual a posteriori error estimation (see e.g. [9]).

Lemma 7.3. Let $\phi_{K}$ be the solution of (56) and let $\bar{\phi}_{K}$ be its mean value over the triangle $K$. Then the following estimate holds:

$$
\begin{aligned}
\mathcal{B}_{K}\left(\phi_{K}-\bar{\phi}_{K}\right) \preceq & \quad m\left\|\phi_{K}\right\| \|_{K}\left(\min \left(h_{\min , K}, \kappa^{-1}\right)\|r\|_{L_{2}(K)}\right. \\
& \left.+\sum_{\gamma \subset \partial K} h_{\min , K}^{1 / 2}\left(\frac{|\gamma|}{|K|}\right)^{1 / 2} \min \left(h_{\min , K}, \kappa^{-1}\right)^{1 / 2}\left\|g_{K}-\frac{\partial u_{X}}{\partial n_{K}}\right\|_{L_{2}(\gamma)}\right) .
\end{aligned}
$$

Proof. We use the definition of the residual functional (50) and apply subsequently the Cauchy-Schwarz inequality and the anisotropic approximation properties (41) and (43) obtained in Lemma 4.6:

$$
\begin{aligned}
\mathcal{B}_{K}\left(\phi_{K}-\bar{\phi}_{K}\right)= & \left(f, \phi_{K}-\bar{\phi}_{K}\right)_{K}-B_{K}\left(u_{X}, \phi_{K}-\bar{\phi}_{K}\right)+\int_{\partial K} g_{K}\left(\phi_{K}-\bar{\phi}_{K}\right) \mathrm{d} s \\
= & \left(r, \phi_{K}-\bar{\phi}_{K}\right)_{K}+\int_{\partial K}\left(g_{K}-\frac{\partial u_{X}}{\partial n_{K}}\right)\left(\phi_{K}-\bar{\phi}_{K}\right) \mathrm{d} s \\
\leq & \|r\|_{L_{2}(K)}\left\|\phi_{K}-\bar{\phi}_{K}\right\|_{L_{2}(K)}+\sum_{\gamma \subset \partial K}\left\|g_{K}-\frac{\partial u_{X}}{\partial n_{K}}\right\|_{L_{2}(\gamma)}\left\|\phi_{K}-\bar{\phi}_{K}\right\|_{L_{2}(\gamma)} \\
\preceq & m\left\|\phi_{K}\right\|_{K} \min \left(h_{\min , K}, \kappa^{-1}\right)\|r\|_{L_{2}(K)} \\
& \quad+{ }_{m} \mid\left\|\phi_{K}\right\|\left\|_{K} \sum_{\gamma \subset \partial K} h_{\min , K}^{1 / 2}\left(\frac{|\gamma|}{|K|}\right)^{1 / 2} \min \left(h_{\min , K}, \kappa^{-1}\right)^{1 / 2}\right\| g_{K}-\frac{\partial u_{X}}{\partial n_{K}} \|_{L_{2}(\gamma)}
\end{aligned}
$$

which completes the proof.

Lemma 7.4. Let $\phi_{K}$ be the solution to (56). Then the following estimate holds:

$$
m\left|\left\|\phi_{K} \mid\right\|_{K} \preceq \min \left(h_{\min , K}, \kappa^{-1}\right)\|r\|_{L_{2}(K)}+\sum_{\gamma \subset \partial K} h_{\min , K}^{1 / 2}\left(\frac{|\gamma|}{|K|}\right)^{1 / 2} \min \left(h_{\min , K}, \kappa^{-1}\right)^{1 / 2}\left\|g_{K}-\frac{\partial u_{X}}{\partial n_{K}}\right\|_{L_{2}(\gamma)}\right.
$$

Proof. We represent $m \mid\left\|\phi_{K}\right\|_{K}^{2}$ as a sum of two terms:

$$
\left.{ }_{m}\left|\| \phi_{K}\right|\right|_{K} ^{2}={ }_{m} B_{K}\left(\phi_{K}, \phi_{K}\right)=\mathcal{B}_{K}\left(\phi_{K}\right)=\mathcal{B}_{K}\left(\phi_{K}-\bar{\phi}_{K}\right)+\mathcal{B}_{K}\left(\bar{\phi}_{K}\right)
$$

In Lemma 7.3 we constructed already the estimate from above for the first term. We proceed estimating the second term analogously to the proof of Theorem 5.2. 
Consider the case $\kappa h_{\min , K} \succeq 1$. Thus, the second assertion of Lemma 5.1 holds, i.e.

$$
\begin{aligned}
\mathcal{B}_{K}\left(\bar{\phi}_{K}\right) & \preceq m\left|\left\|\phi_{K} \mid\right\|_{K}\left(\kappa^{-1}\|r\|_{L_{2}(K)}+\sum_{\gamma \subset \partial K}\left(\frac{|\gamma|}{|K|}\right)^{1 / 2} \kappa^{-1}\left\|g_{K}-\frac{\partial u_{X}}{\partial n_{K}}\right\|_{L_{2}(\gamma)}\right)\right. \\
\preceq & m\left|\| \phi _ { K } | \| _ { K } \left(\min \left(h_{\min , K}, \kappa^{-1}\right)\|r\|_{L_{2}(K)}\right.\right. \\
& \left.+\sum_{\gamma \subset \partial K} h_{\min , K}^{1 / 2}\left(\frac{|\gamma|}{|K|}\right)^{1 / 2} \min \left(h_{\min , K}, \kappa^{-1}\right)^{1 / 2}\left\|g_{K}-\frac{\partial u_{X}}{\partial n_{K}}\right\|_{L_{2}(\gamma)}\right),
\end{aligned}
$$

where we used $\kappa \succeq h_{\min , K}^{-1}$.

The remaining elements satisfy $\kappa h_{\min , K} \ll 1$. With the same arguments as in the proof of Theorem 5.2 we verify that for these elements (55) holds, and thus, the second term in (60) vanishes.

Summing up the contributions from the two terms on the right hand side of representation (60) and dividing the concluding inequality by $m \mid\left\|\phi_{K}\right\|_{K}$ we get the result claimed.

In order to prove the main theorem of this section we need some additional elementary facts.

Lemma 7.5. Let $\phi \in P_{1}([-1,1])$ be a linear function. For the $L_{2}$-projection operator $I: P_{1}([-1,1]) \rightarrow$ span $\left\{1-x^{2}, x\left(1-x^{2}\right)\right\}$ the following inequality holds:

$$
\sqrt{\frac{6}{5}}\|I \phi\|_{L_{2}([-1,1])} \leq\|\phi\|_{L_{2}([-1,1])} \leq \sqrt{\frac{10}{7}}\|I \phi\|_{L_{2}([-1,1])} .
$$

Proof. Denote $\psi_{1}:=1-x$ and $\psi_{2}:=1+x$. The desired constants are the square roots of the maximal and minimal eigenvalues of the eigenvalue problem $A \cdot \mathbf{x}=\lambda B \cdot \mathbf{x}$, where $A=\left\{\int_{-1}^{1} \psi_{i} \psi_{j} \mathrm{~d} x\right\} \in \mathbb{R}^{2 \times 2}$, $B=\left\{\int_{-1}^{1} I \psi_{i} I \psi_{j} \mathrm{~d} x\right\} \in \mathbb{R}^{2 \times 2}$ and $\mathbf{x} \in \mathbb{R}^{2}$.

Lemma 7.6. Let $\gamma$ be an edge of a triangle $K$. We define the operator $I_{\gamma}: P_{1}(\gamma) \rightarrow \operatorname{span}\left\{b_{\gamma, \delta_{\gamma}}, s_{\gamma, \delta_{\gamma}}\right\}$ so that for any function $\phi \in P_{1}(\gamma)$ the restriction of the resulting function $\left.I_{\gamma} \phi\right|_{\gamma}$ to the edge $\gamma$ is the $L_{2}$ projection of $\phi$ to the space $\operatorname{span}\left\{\left.b_{\gamma, \delta_{\gamma}}\right|_{\gamma},\left.s_{\gamma, \delta_{\gamma}}\right|_{\gamma}\right\}$. In other words, we project a function $\phi$ onto the space of two functions on the edge $\gamma$ and then take the corresponding constant to produce the function $I_{\gamma} \phi$ inside the triangle $K$. For the operator $I_{\gamma}$ and any function $\phi \in P_{1}(\gamma)$ the following estimate holds:

$$
\sqrt{\frac{6}{5}}\left\|I_{\gamma} \phi\right\|_{L_{2}(\gamma)} \leq\|\phi\|_{L_{2}(\gamma)} \leq \sqrt{\frac{10}{7}}\left\|I_{\gamma} \phi\right\|_{L_{2}(\gamma)} .
$$

Proof. The functions $1-x^{2}$ and $x\left(1-x^{2}\right)$ coincide with the functions $\left.b_{\gamma, \delta_{\gamma}}\right|_{\gamma}$ and $\left.s_{\gamma, \delta_{\gamma}}\right|_{\gamma}$ whenever $\gamma=[-1,1]$. Thus, the assertion follows from Lemma 7.5 and standard transformation techniques.

We proceed with the main result of this section, that guarantees the estimate from above for the solution of the local problem (56).

Theorem 7.7. Let $\phi_{K}$ and $\widetilde{\phi}_{K}$ be the solutions to the problems (56) and (58) respectively. Then:

$$
{ }_{m}\left|\left\|\phi_{K}\left|\left\|_{K} \preceq{ }_{m}\right\| \widetilde{\phi}_{K}\right|\right\|_{K}+\min \left(h_{\min , K}, \kappa^{-1}\right)\|r-\bar{r}\|_{L_{2}(K)} .\right.
$$

Proof. We will essentially use the estimate of Lemma 7.4 and bound the terms on the right hand side by $m\|\| \widetilde{\phi}_{K} \mid \|_{K}$. 
Performing the partial integration we can rewrite the finite dimensional local problem (58) as

$$
\begin{aligned}
{ }_{m} B_{K}\left(\widetilde{\phi}_{K}, v\right) & =(r, v)_{K}+\int_{\partial K}\left(g_{K}-\frac{\partial u_{X}}{\partial n_{K}}\right) v \mathrm{~d} s \\
& =\left(r_{K}, v\right)_{K}+\int_{\partial K}\left(g_{K}-\frac{\partial u_{X}}{\partial n_{K}}\right) v \mathrm{~d} s+\left(r-r_{K}, v\right)_{K} .
\end{aligned}
$$

Since (61) holds for all $v \in V_{b}(K)$, we substitute $v:=b_{K} \bar{r}$,

$$
\int_{K} b_{K} \bar{r}^{2} \mathrm{~d} x={ }_{m} B_{K}\left(\phi_{K}, b_{K} \bar{r}\right)-\left(r-r_{K}, b_{K} \bar{r}\right)_{K} .
$$

Using (23), we obtain with the aid of Cauchy-Schwarz inequality

$$
\|\bar{r}\|_{L_{2}(K)}^{2} \preceq{ }_{m}\left\|\widetilde{\phi}_{K}\left|\left\|_{K m}\right\|\right|\right\| b_{K} \bar{r}\left\|_{K}+\right\| r-\bar{r}\left\|_{L_{2}(K)}\right\| b_{K} \bar{r} \|_{L_{2}(K)} .
$$

Applying estimates (24) and (28) of Lemma 4.2 and dividing both parts by $\min \left(h_{\min , K}, \kappa^{-1}\right)^{-1}\|\bar{r}\|_{L_{2}(K)}$, we get

$$
\min \left(h_{\min , K}, \kappa^{-1}\right)\|\bar{r}\|_{L_{2}(K)} \preceq m\|\| \widetilde{\phi}_{K}\left\|_{K}+\min \left(h_{\min , K}, \kappa^{-1}\right)\right\| r-\bar{r} \|_{L_{2}(K)},
$$

or, with the aid of the triangle inequality we derive the upper bound for the term involving the element residual:

$$
\min \left(h_{\min , K}, \kappa^{-1}\right)\|r\|_{L_{2}(K)} \preceq m\|\| \widetilde{\phi}_{K}\left\|_{K}+\min \left(h_{\min , K}, \kappa^{-1}\right)\right\| r-\bar{r} \|_{L_{2}(K)} .
$$

Suppose $\gamma \subset \partial K \backslash \partial \Omega$ is one of the edges of $K$, which is not a Dirichlet edge. We choose now $v:=I_{\gamma}\left(g_{K}-\frac{\partial u_{X}}{\partial n_{K}}\right) \in$ $V_{b}(K)$ in (61), where the operator $I_{\gamma}$ was defined in Lemma 7.6.

$$
{ }_{m} B_{K}\left(\widetilde{\phi}_{K}, I_{\gamma}\left(g_{K}-\frac{\partial u_{X}}{\partial n_{K}}\right)\right)=\left(r, I_{\gamma}\left(g_{K}-\frac{\partial u_{X}}{\partial n_{K}}\right)\right)_{K}+\int_{\partial K}\left(g_{K}-\frac{\partial u_{X}}{\partial n_{K}}\right) \cdot I_{\gamma}\left(g_{K}-\frac{\partial u_{X}}{\partial n_{K}}\right) \mathrm{d} s .
$$

Since $I_{\gamma}$ is the $L_{2}$-projection operator over $\gamma$, we rewrite the last equality in the form:

$$
\left\|I_{\gamma}\left(g_{K}-\frac{\partial u_{X}}{\partial n_{K}}\right)\right\|_{L_{2}(\gamma)}^{2}={ }_{m} B_{K}\left(\widetilde{\phi}_{K}, I_{\gamma}\left(g_{K}-\frac{\partial u_{X}}{\partial n_{K}}\right)\right)-\left(r, I_{\gamma}\left(g_{K}-\frac{\partial u_{X}}{\partial n_{K}}\right)\right)_{K},
$$

and utilizing the Cauchy-Schwarz inequality, we obtain:

$$
\left\|I_{\gamma}\left(g_{K}-\frac{\partial u_{X}}{\partial n_{K}}\right)\right\|_{L_{2}(\gamma)}^{2} \leq{ }_{m}\|\widetilde{\phi}\|\left\|_{K m}\right\| I_{\gamma}\left(g_{K}-\frac{\partial u_{X}}{\partial n_{K}}\right)\left\|_{K}+\right\| r\left\|_{L_{2}(K)}\right\| I_{\gamma}\left(g_{K}-\frac{\partial u_{X}}{\partial n_{K}}\right) \|_{L_{2}(K)} .
$$

Evaluating (36) and (37) of Lemma 4.3 together with Lemma 7.6, we complete the proof.

To complete the discussion of the equilibrated residual method it only remains to give a definition for the error estimator that can be used in practical computations and to give the resulting bounds provided by the preceding theory.

Definition 7.8. We define the local estimator of the equilibrated residual method and the local higher order term corresponding to the element $K$ by

$$
\begin{aligned}
\eta_{E R, K} & :=m\left\|\widetilde{\phi}_{K}\right\|_{K}, \\
\zeta_{K} & :=\min \left(h_{\min , K}, \kappa^{-1}\right)\|r-\bar{r}\|_{L_{2}(K)},
\end{aligned}
$$




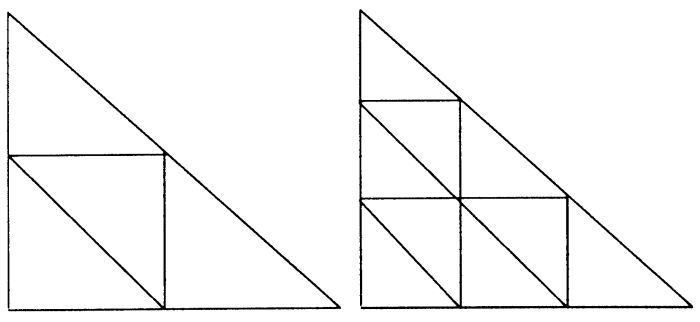

FiguRE 7 . Triangle subdivisions. $n=2$ and $n=3$, respectively.

with the global counterparts

$$
\begin{aligned}
\eta_{E R} & :=\left(\sum_{K \in \mathcal{T}} \eta_{E R, K}^{2}\right)^{1 / 2} \text { and } \\
\zeta & :=\left(\sum_{K \in \mathcal{T}} \zeta_{K}^{2}\right)^{1 / 2},
\end{aligned}
$$

respectively.

Combining the bounds from Theorems $6.1,6.2,7.1$ and 7.7 we arrive at the robustness result for the error estimator defined above.

Theorem 7.9. In notation of Definition 7.8 the upper and lower error bounds hold

$$
\begin{aligned}
\|e\|^{2} & \preceq m_{1}^{2}(e, \mathcal{T})\left(\eta_{E R}^{2}+\zeta^{2}\right), \\
\eta_{E R, K}^{2} & \preceq\|\| \|_{\omega_{K}}^{2}+\sum_{K \subset \omega_{K}} \zeta_{K}^{2} .
\end{aligned}
$$

This theorem is the final result of this work and guarantees the reliability and efficiency of the estimator. The bounds are in accordance with those provided by Kunert [21] for the Dirichlet local problem error estimator for the singularly perturbed reaction-diffusion equation.

\section{NUMERICAL EXPERIMENTS}

In the previous section we gave an example for the bases for the local problem (56) consisting of the very specially squeezed functions. We have good experience, however, in solving this problem with a finite element method, where we choose the nodal basis corresponding to a division of the triangles into $n^{2}$ parts, see Figure 7 .

Let us consider the 2D model problem

$$
-\Delta u+\kappa^{2} u=0 \text { in } \Omega:=[0,1]^{2}, \quad u=u_{0} \text { on } \partial \Omega .
$$

Prescribe the exact solution

$$
u=\mathrm{e}^{-\kappa x}+\mathrm{e}^{-\kappa y}
$$

which displays typical boundary layers along the sides $x=0$ and $y=0$. The Dirichlet boundary data $u_{0}$ are chosen accordingly.

We use a sequence of finite element meshes generated by the algorithm described in [7]. The idea of adaptive procedure is that the choice of a refinement direction is done according to the components of energy norm of 


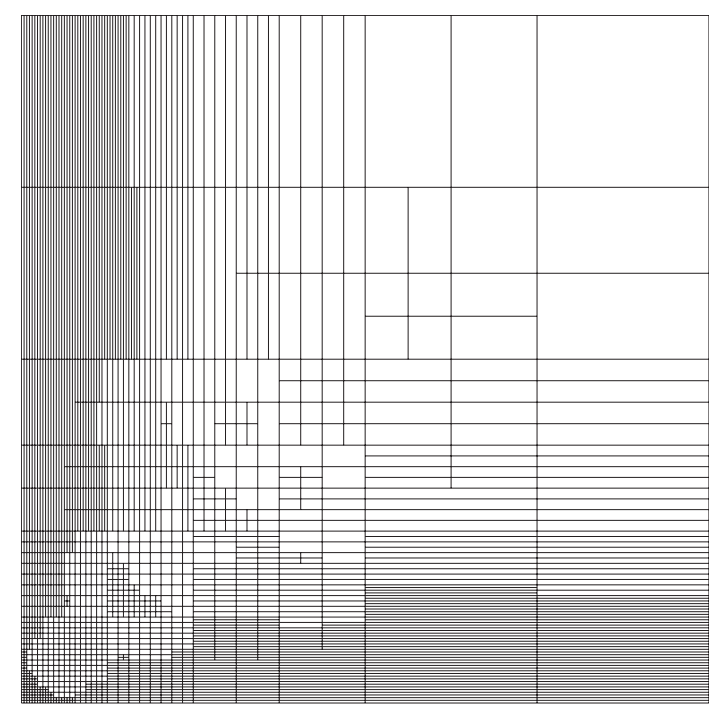

Figure 8. Mesh refinement.

\begin{tabular}{|c|c|c|r|r|}
\hline Iteration N & Unknowns N & Maximal aspect ratio & $\frac{\text { AB error }}{\text { exact err }}$ & $\frac{\text { AB er(mod) }}{\text { exact err }}$ \\
\hline \hline 1 & 25 & 71 & 1.392 & 1.102 \\
2 & 51 & 71 & 1.301 & 0.896 \\
3 & 97 & 71 & 1.565 & 0.962 \\
4 & 116 & 142 & 1.903 & 1.032 \\
5 & 157 & 285 & 2.457 & 1.094 \\
6 & 217 & 571 & 3.151 & 1.153 \\
7 & 382 & 1142 & 4.264 & 1.169 \\
8 & 714 & 2284 & 5.955 & 1.167 \\
9 & 1481 & 4568 & 8.903 & 1.157 \\
10 & 3274 & 9137 & 14.475 & 1.130 \\
11 & 6847 & 18273 & 23.967 & 1.112 \\
12 & 15187 & 36547 & 44.111 & 1.090 \\
13 & 35536 & 73095 & 81.237 & 1.062 \\
14 & 106819 & 146191 & 138.711 & 1.005 \\
\hline
\end{tabular}

TABLE 1. Results for $n=4, \kappa=1000$. The fourth column represents the ratio between the Ainsworth and Babuška estimator and the energy norm of the true solution, while the fifth column represents the similar ratio for the estimator defined in the current work.

an error $\left\|\frac{\partial e}{\partial x}\right\|_{L_{2}(K)},\left\|\frac{\partial e}{\partial y}\right\|_{L_{2}(K)}$, and $\kappa^{2}\|e\|_{L_{2}(K)}$. One of the resulting meshes of this program is displayed in Figure 8.

Tables 1 and 2 show the behavior of the estimators in the singularly perturbed case on anisotropic meshes. We observe that the new error estimator is robust while the original one overestimates the true error when the aspect ratio is large enough. 


\begin{tabular}{|c|c|c|r|r|}
\hline Iteration N & Unknowns N & Maximal aspect ratio & $\frac{\text { AB error }}{\text { exact err }}$ & $\frac{\text { AB er(mod) }}{\text { exact err }}$ \\
\hline \hline 1 & 25 & 541 & 2.665 & 2.150 \\
2 & 51 & 541 & 2.177 & 1.587 \\
3 & 120 & 541 & 1.802 & 1.198 \\
4 & 143 & 541 & 1.995 & 1.187 \\
5 & 192 & 1083 & 2.410 & 1.243 \\
6 & 217 & 2167 & 3.062 & 1.302 \\
7 & 283 & 4335 & 4.273 & 1.392 \\
8 & 446 & 8669 & 6.207 & 1.436 \\
9 & 814 & 17339 & 9.748 & 1.405 \\
10 & 1553 & 34679 & 16.646 & 1.411 \\
11 & 3053 & 69359 & 30.149 & 1.433 \\
12 & 5809 & 138718 & 54.363 & 1.429 \\
13 & 11357 & 277436 & 101.47 & 1.420 \\
14 & 23376 & 554873 & 211.02 & 1.407 \\
15 & 104916 & 1109745 & 423.10 & 1.383 \\
\hline
\end{tabular}

TABLE 2. Results for $n=4, \kappa=10000$.

\section{Summary AND ADDitional REMARKS}

We consider the singularly perturbed reaction-diffusion equation $-\Delta u+\kappa^{2} u=f$. This work has been aiming at a posteriori equilibrated residual-like error estimators suitable for anisotropic triangular grids.

The Ainsworth-Babuška estimator is shown to be reliable in the anisotropic case. Unfortunately, the lower error bound fails on anisotropic meshes.

The introduced modification leads to an estimator which is robust with respect to the anisotropy of the mesh as well as to the singular perturbation. Upper and lower error bounds are proved. The factor which made the original error estimator fail does not appear in the lower bound any more, which leads to the efficiency of the modified estimator. The upper error bound of the modified estimator contains the factor $m_{1}(e, \mathcal{T})$ which is in accordance with the results made by Kunert in [21]. Furthermore, an appropriate basis for the approximate solution of the local problem is provided and the whole method is shown not to be affected by this approximation.

The numerical experiments verify the theory. The modified estimator yields a useful and reliable error bound not only in an asymptotic sense but also for meshes with moderate number of elements.

Remark 9.1. All the proofs are suitable for 3D case. The only two questions we should answer are about the topology matrices and the minimum energy extension of the first-order basis function. For the minimum energy extension of the first-order basis function we construct the approximation by analogy with Section 3.3. A point $D$ in this case may be chosen on the intersection line of the bisection planes of the corresponding cone with the distance $1 / \kappa$ from each face. The topology matrices are constructed in the way analogous to Section 3 , but seem to be much more complicated.

Remark 9.2. Neumann boundary conditions can be also considered as well as quadrilateral elements. In both cases an additional term corresponding to the face residual appears in the lower bound for the error:

$$
\begin{aligned}
\left\|\phi_{K}\right\|_{K} & \preceq\|e\|_{\tilde{K}}+\min \left(h_{\min , K}, \kappa^{-1}\right)\|r-\bar{r}\|_{L_{2}(\widetilde{K})} \\
& +\sum_{\gamma \subset \widetilde{K}} \min \left(h_{\min , K}, \kappa^{-1}\right)^{1 / 2}\|R-\bar{R}\|_{L_{2}(\gamma)} .
\end{aligned}
$$

Remark 9.3. If we solve our FEM problem with polynomials of $p$-th order then we have to talk about the $p$-th order equilibration. For details see [3]. 
Remark 9.4. Consider the problem in another formulation used by some authors (see $[6,8,16,21]$ ), namely

$$
-\varepsilon^{2} \Delta u+u=f \text { in } \Omega \subset \mathbb{R}^{2}, \quad u=0 \text { on } \partial \Omega .
$$

The estimator remains the same as well as its upper error bound:

$$
\|\mid\|^{2} \leq m_{1}(e, \mathcal{T})^{2} \sum_{K \in \mathcal{T}} m\left\|\phi_{K}\right\|_{K}^{2}
$$

The lower error bound changes to the following:

$$
{ }_{m}\left\|\phi _ { K } \left|\left\|_{K} \preceq \mid\right\| e\left\|_{\widetilde{K}}+\min \left(h_{\min , K} \varepsilon^{-1}, 1\right)\right\| r-\bar{r} \|_{L_{2}(\widetilde{K})} .\right.\right.
$$

We should note that this is exactly the same estimate as for another error estimator introduced by Kunert in $[21]$.

Acknowledgements. I would like to thank the Deutsche Forschungsgemeinschaft, SFB393, for their support. I would also like to express my sincere thanks to Thomas Apel, Gerd Kunert and Cornelia Pester for the fruitful discussions.

\section{REFERENCES}

[1] M. Ainsworth and I. Babuška, Reliable and robust a posteriori error estimation for singularly perturbed reaction-diffusion problems. SIAM J. Numer. Anal. 36 (1999) 331-353 (electronic). See also Corrigendum at http://www.maths.strath.ac.uk/ aas98107/papers.html.

[2] M. Ainsworth and J.T. Oden, A unified approach to a posteriori error estimation using element residual methods. Numer. Math. 65 (1993) 23-50.

[3] M. Ainsworth and J.T. Oden, A Posteriori Error Estimation in Finite Element Analysis. Wiley (2000).

[4] T. Apel, Anisotropic interpolation error estimates for isoparametric quadrilateral finite elements. Computing 60 (1998) 157174 .

[5] T. Apel, Treatment of boundary layers with anisotropic finite elements. Z. Angew. Math. Mech. (1998).

[6] T. Apel, Anisotropic finite elements: local estimates and applications. B.G. Teubner, Stuttgart (1999).

[7] T. Apel, S. Grosman, P.K. Jimack and A. Meyer, A new methodology for anisotropic mesh refinement based upon error gradients. Appl. Numer. Math. 50 (2004) 329-341.

[8] T. Apel and G. Lube, Anisotropic mesh refinement for a singularly perturbed reaction diffusion model problem. Appl. Numer. Math. 26 (1998) 415-433.

[9] I. Babuška and W. Rheinboldt, A posteriori error estimates for the finite element method. Int. J. Numer. Meth. Eng. 12 (1978) 1597-1615.

[10] R. Bank and A. Weiser, Some a posteriori error estimators for elliptic partial differential equations. Math. Comp. 44 (1985) 283-301.

[11] H. Bufler and E. Stein, Zur Plattenberechnung mittels finiter Elemente. Ingenier Archiv 39 (1970) 248-260.

[12] P.G. Ciarlet, The finite element method for elliptic problems. North-Holland Publishing Co., Amsterdam. Studies in Mathematics and its Applications, Vol. 4, (1978).

[13] M. Dobrowolski, S. Gräf and C. Pflaum, On a posteriori error estimators in the infinte element method on anisotropic meshes. Electron. Trans. Numer. Anal. 8 (1999) 36-45.

[14] S. Grosman, The robustness of the hierarchical a posteriori error estimator for reaction-diffusion equation on anisotropic meshes. SFB393-Preprint 2, Technische Universität Chemnitz, SFB 393 (Germany), (2004).

[15] R. Hagen, S. Roch, and B. Silbermann, $C^{*}$-algebras and numerical analysis. Marcel Dekker Inc., New York (2001).

[16] H. Han and R.B. Kellogg, Differentiability properties of solutions of the equation $-\epsilon^{2} \delta u+r u=f(x, y)$ in a square. SIAM J. Math. Anal. 21 (1990) 394-408.

[17] G. Kunert, A posteriori error estimation for anisotropic tetrahedral and triangular finite element meshes. Logos Verlag, Berlin, 1999. Also PhD thesis, TU Chemnitz, http://archiv.tu-chemnitz.de/pub/1999/0012/index.html.

[18] G. Kunert, An a posteriori residual error estimator for the finite element method on anisotropic tetrahedral meshes. Numer. Math. 86 (2000) 471-490. 
[19] G. Kunert, A local problem error estimator for anisotropic tetrahedral finite element meshes. SIAM J. Numer. Anal. 39 (2001) $668-689$.

[20] G. Kunert, Robust a posteriori error estimation for a singularly perturbed reaction-diffusion equation on anisotropic tetrahedral meshes. Adv. Comput. Math. 15 (2001) 237-259.

[21] G. Kunert, Robust local problem error estimation for a singularly perturbed problem on anisotropic finite element meshes. ESAIM: M2AN 35 (2001) 1079-1109.

[22] G. Kunert and R. Verfürth, Edge residuals dominate a posteriori error estimates for linear finite element methods on anisotropic triangular and tetrahedral meshes. Numer. Math. 86 (2000) 283-303.

[23] P. Ladevèze and D. Leguillon, Error estimate procedure in the finite element method and applications. SIAM J. Numer. Anal. 20 (1983) 485-509.

[24] K.G. Siebert, An a posteriori error estimator for anisotropic refinement. Numer. Math. 73 (1996) 373-398.

[25] R. Verfürth, A review of a posteriori error estimation and adaptive mesh-refinement techniques. Wiley-Teubner Series Advances in Numerical Mathematics. Chichester: John Wiley \& Sons. Stuttgart: B.G. Teubner (1996).

[26] M. Vogelius and I. Babuška, On a dimensional reduction method. I. The optimal selection of basis functions. Math. Comp. 37 (1981) $31-46$.

To access this journal online:

www.edpsciences.org 